NOTE TO USERS

This reproduction is the best copy available.

(B)

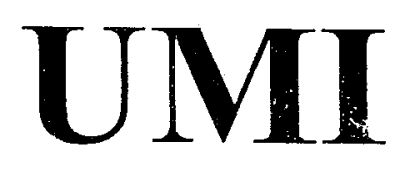

Reproduced with permission of the copyright owner. Further reproduction prohibited without permission. 
Reproduced with permission of the copyright owner. Further reproduction prohibited without permission. 


\title{
MODEL-BASED SEGMENTATION AND ANALYSIS OF KNEE SOUND SIGNALS
}

\author{
By \\ Farhana Parveen

\begin{abstract}
A PROJECT
SUBMITTED IN PARTIAL FULFILLMENT OF THE

REQUIREMENTS FOR THE DEGREE OF MASTER OF ENGINEERING

$\mathrm{AT}$

RYERSON UNIVERSITY

350 VICTORIA STREET, TORONTO, ONTARIO, CANADA

JANUARY 2004
\end{abstract}

(C) Copyrimbt by Farhana Parveen, 2004 
UMI Number: EC52945

\section{INFORMATION TO USERS}

The quality of this reproduction is dependent upon the quality of the copy submitted. Broken or indistinct print, colored or poor quality illustrations and photographs, print bleed-through, substandard margins, and improper alignment can adversely affect reproduction.

In the unlikely event that the author did not send a complete manuscript and there are missing pages, these will be noted. Also, if unauthorized copyright material had to be removed, a note will indicate the deletion.

(B)

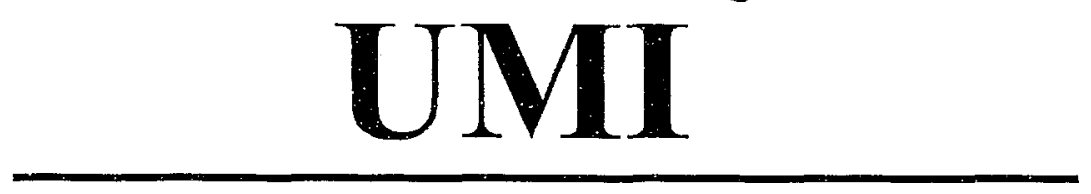

UMI Microform EC52945

Copyright 2008 by ProQuest LLC.

All rights reserved. This microform edition is protected against unauthorized copying under Title 17, United States Code.

ProQuest LLC

789 E. Eisenhower Parkway

PO Box 1346

Ann Arbor, Ml 48106-1346 
Reproduced with permission of the copyright owner. Further reproduction prohibited without permission. 
To My parents, Monjur and Kishan

Reproduced with permission of the copyright owner. Further reproduction prohibited without permission. 


\title{
Table of Contents
}

\author{
Table of Contents
}

Abstract $\quad$ ix

Acknowledgements $\quad x$

1 Introduction 1

1.1 Anatomy of the Normal Knee Joint . . . . . . . . . . . . . . . . . . 1

1.2 Knee-Joint Pathology: Osteoarthritis . . . . . . . . . . . . . . 2

1.3 Review of Non-Invasive Diagnosis of Osteoarthritis: . . . . . . . . 5

1.4 An Introduction to Knee Sound Signals . . . . . . . . . . . . . . . . 7

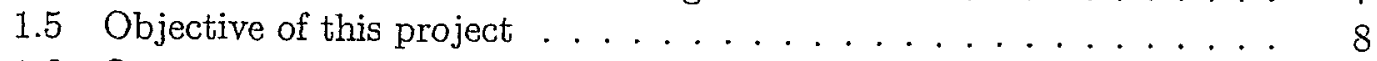

1.6 Organization of the Report . . . . . . . . . . . . . 9

2 Related Works 10

2.1 Previous Research . . . . . . . . . . . . . . . . . . . . 10

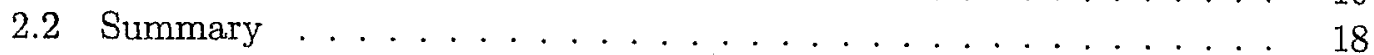

3 Segmentation Based Analysis of VAG $\quad 19$

3.1 Different Techniques of Adaptive Segmentation .......... 20

3.2 Initial Segmentation (Fixed Width) . . . . . . . . . . . . . 22

3.3 Model-Based Adaptive Segmentation of VAG Signals . . . . . . . . . . . . 24

3.4 Finding the Model Order . . . . . . . . . . . . . . . . . . . 27

3.5 LPC Modelling of the Signal . . . . . . . . . . . . . . . . . 28

4 Pattern Classification 31

4.1 Different Pattern Classification Techniques . . . . . . . . . . . . . 32

4.1.1 Principal Component Analysis . . . . . . . . . . . . . . . . 32

4.1 .2 Genetic Algorithm ................. 33 
4.1 .3 Logistic Regression . . . . . . . . . . . . . . . . . . . 34

4.1.4 Maximum Likelihood Estimation . . . . . . . . . . . 34

4.1.5 Decision Trees . . . . . . . . . . . . . . . . 35

4.1.6 Linear Discriminant Analysis . . . . . . . . . . . . . . . . 35

4.2 Pattern Analysis of VAG . . . . . . . . . . . . . 40

5 Results and Conclusion $\quad 42$

5.1 Motivation for Our strategy . . . . . . . . . . . . . . . 42

5.2 Results of Localization of Pathology . . . . . . . . . . . . 43

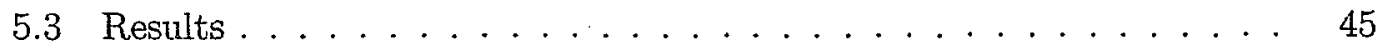

5.4 Conclusion . . . . . . . . . . . . . . . . . . 47

5.5 Possible Future Work Direction . . . . . . . . . . . . 47

$\begin{array}{ll}\text { A } & 48\end{array}$

$\begin{array}{ll}\mathrm{B} & 53\end{array}$

$\begin{array}{ll}\text { Bibliography } & 56\end{array}$

vi

Reproduced with permission of the copyright owner. Further reproduction prohibited without permission. 


\section{List of Tables}

5.1 Starting and End point of each segment after doing the adaptive segment 44

5.2 Classification results using LDA . . . . . . . . . . . . . . . 46

5.3 Classification using leave-one-out method . . . . . . . . . . . . 47

vii

Reproduced with permission of the copyright owner. Further reproduction prohibited without permission. 


\section{List of Figures}

1.1 Anatomy of the human knee joint $[18] \ldots \ldots . \ldots 2$

1.2 Osteoarthritis on human knee joint $[19] \ldots \ldots . \ldots . \ldots 3$

2.1 the left one shows The arrow points to a click Heard at $20^{\circ}$ extension $0^{\circ}$ is the Fully extended position and the right one shows the Arrows point to clicks and grinding sounds heard during auscultation [4] . . I 15

3.1 VAG when a person moving his leg from $135^{\circ}-0^{0}$ extension . . . . 23

3.2 Fixed segmentation of the signal shown in Figure $3.1 \ldots 23$

3.3 Adaptive segmentation of the signal . . . . . . . . . . . 25

3.4 Averaged signal for finding the number of peaks . . . . . . . . . . 28

4.1 Flowchart of VAG Analysis . . . . . . . . . . . . . . . 41

5.1 Adaptive segmentation of the signal showing the localization . . . . . 43

viii

Reproduced with permission of the copyright owner. Further reproduction prohibited without permission. 


\section{TITLE: MODEL BASED SEGMENTATION AND ANALYSIS OF KNEE SOUND SIGNAL}

\section{Abstract}

The motivation of the work is to develop a signal processing methodology for noninvasive diagnosis of knee osteoarthritis in an early stage. The sound signal that is emitted from knee when it moves is called Vibroarthrographic (VAG) signal. Analysis of this sound signal will help in diagnosis of the knee joint problems.

In this project a model based approach for segmenting the VAG signals, followed by feature extraction and classification is proposed. This could be used to get some indication whether the signal is from a normal knee or from an abnormal knee. The proposed scheme also has the capability for finding the depth of severity of the damage and it can also localize the angle range of the knee swing, where the damage has occurred. As a result, the project gave an accuracy of $70.4 \%$ with leave-one-out method.

After doing the classification using the segments, finally it has been calculated how many segments from each signal has been correctly identified. A total of $30 \mathrm{knee}$ sound signals from normal and abnormal knees has been used in this work and out of that 26 signals has been classified properly (either normal or abnormal) and 4 signals got misclassified. with a successful classification accuracy of $86.7 \%$. 


\section{Acknowledgements}

I would like to take the opportunity to thank the people who have offered technical insight, inspiration towards the successful completion of this project.

I want to give the major credit to Dr. Krishnan, without his cordial guidance it was impossible to finish this work. During my whole period of doing this Masters program, his continuous help and inspiration was incomparable.

I am grateful to my husband Monjur for giving me his sole support to finish this work.

Also I want to thanks to all my classmates and associates of the graduate studies department.

Toronto, Ontario

Farhana Parveen

January 14, 2004 


\section{Chapter 1}

\section{Introduction}

\subsection{Anatomy of the Normal Knee Joint}

Knee joint is the most complex joint in human structure. Flexion and extension are the main knee movements although some rotation occurs when the knee is flexed. The sound emitted from the knee when it moves, called the Vibroarthrographic (VAG) signal. Here (Figure 1.1) is a brief description of the normal human knee joint for both flexion and extension position.

The knee is a "hinge type" joint, which is formed by two bones held together by flexible ligaments. The bones are the femur (thigh bone) and the tibia (shin bone). The knee cap (patella) also forms part of the knee joint. It glides over the end of the femur as the knee bends. The moving parts of a normal knee are covered with a layer of articular cartilage, which is a white smooth substance about $1 / 4$ of an inch thick on the patella and 1/8 of an inch thick on the femur and tibia. An x-ray of the knee normally shows space (the "joint space") between the femur and the tibia as well as between the femur and the patella. This is not empty space but represents the cartilage (which does not show up on $\mathrm{x}$-rays). The smooth, cartilage-covered surfaces 


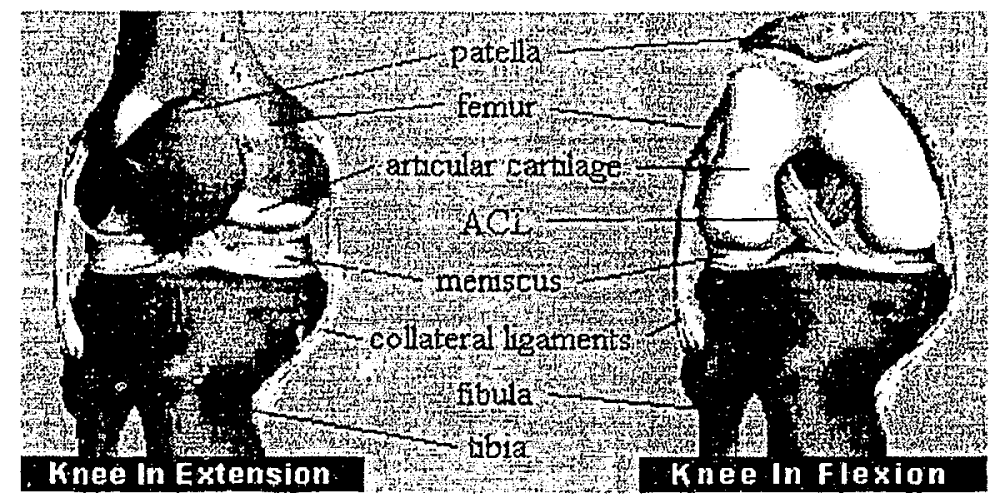

Figure 1.1: Anatomy of the human knee joint [18]

of the knee move on each other with very little friction in the normal joint. In the normal lnee the "joint space" is approximately $1 / 4$ of an inch wide and fairly even in outline.

\subsection{Knee-Joint Pathology: Osteoarthritis}

There are several knee-joint pathologies and the osteoarthritis is the most common one. The term "arthritis" literally means inflammation of a joint, but is generally used. to describe any condition in which there is damage to the cartilage. Inflammation, if present, is in the synovium. The proportion of cartilage damage and synovial inflammation varies with the type and stage of arthritis. Usually the pain early on is due to inflammation. In the later stages, when the cartilage is worn away, most of the pain comes from the mechanical friction of raw bones rubbing on each other.

Knee cartilage can be compared to the tread of an automobile tire, very durable but susceptible to wear over time. Osteoarthritis is a clegenerative process, which 


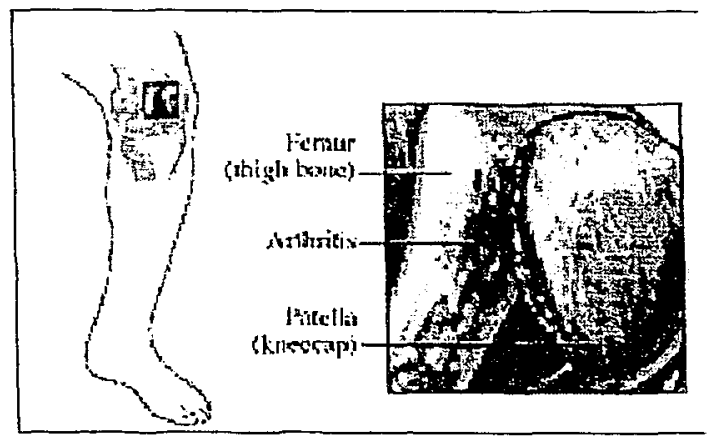

Figure 1.2: Osteoarthritis on human knee joint [19]

results in the wearing out of the joint surface. Over time the joint surface slowly erodes until the underlying bone is exposed. This exposed bone can be painful when the joint moves and bears weight. Osteoarthritis mainly damages the joint cartilage, but there is often some infiammation as well. It usually affects only one or two major joints (ustually in the legs). It does not affect the internal organs. The cause of knee osteoarthritis is not known. It is thought to be simply a process of "wear and tear" in most cases. Some conditions may predispose the knee to osteoarthritis, for example, a previcus fracture that involved the joint, or by lesser injuries that may have torn ligaments or menisci. In osteoarthritis of the knee the cartilage cushion is either thinner than normal (leaving bare spots on the bone), or completely absent. Bare bones grind against each other and cause mechanical pain. Fragments of cartilage floating in the joint may cause inflammation in the joint lining, and this is a second source of pain. X-rays show the "joint space" to be narrowed and irregular in outline. There is no blood test for diagnosing osteoarthritis and its progression.

Many patients have knee pain coming from injury to a meniscal cartilage rather 
than injury to the articular cartilage. The comparison between two categories are as follows:

- The articular cartilage is the cartilage that covers the ends of the bone (similar to the tread on a tire).

- A meniscal cartilage is a disc of cartilage that is actually separate from the femur, the tibia and the patella. There are two such c-shaped meniscal cartilages in the knee.

They are sandwiched between the femur and the tibia. These meniscal cartilages are often injured, particularly during athletics of a meniscal cartilage is torn, it often does not heal and the pieces of the cartilage may become trapped in abnormal positions in the knee causing giving way, fluid on the knee, and pain with certain twisting activities. However, when the articular cartilage has been worn out (as in arthritis), arthroscopy is rarely able to correct the problem and a knee replacement is often needed. Osteoarthritis of the knee often develops in one weight-bearing compartment of the knee, while the other two compartments remain relatively healthy. Since osteoarthritis is a progressive disease, in the short term it can be managed conservatively. Anti-inflammatory drugs, cortisone injections, and physical therapy can delay the need for surgery, but eventually many people require surgical reconstruction of the knee. The traditional approach to knee reconstruction has been a total knee replacement, which replaces all three compartments of the knee. A total knee provides excellent pain relief and has been shown to be very durable. The problem with approach is that it's a expensive procedure. For people who need two or more of their knee compartments resurfaced, total knee replacement is an excellent choice to relieve pain and restore function of the knee. 


\subsection{Review of Non-Invasive Diagnosis of Osteoarthri- tis:}

There are a number of non-invasive diagnostic method for Osteoarthritis (OA) other than the general clinical diagnosis like inspection, function testing and palpation. Few of them are described below [20]:

(a) Plain Radiographs or X-rays: Plain X-rays have been the primary imaging method used to confirm the diagnosis of OA, stage its severity, and follow its progression. Unfortunately, the severity of radiographic findings do not correlate well with the severity of symptoms or the presence of functional disability. Although more than $90 \%$ of people over age 40 have some radiographic evidence of osteoarthritic joint changes, only $30 \%$ have symptoms. This limits the use of radiography, both as a diagnostic tool and as a tool for ongoing assessment of disease progression. Because the plain radiograph is almost always sufficient, most of the following techniques are only used in special circumstances to aid in the diagnosis of OA. For example, these studies can be used to eliminate an infected joint (e.g., radionuclide scintigraphy) or to look for a herniated disc in the back (e.g., magnetic resonance imaging MRI).

(b) Arthrography: This involves the injection of contrast media into a joint. Arthrog$\mathrm{r} \sim$ hy is a relatively time-consuming and invasive procedure, but it may be useful in detecting meniscal injury, loose bodies in the joint cavity, and other local changes in joint structure. It has been mostly replaced by MRI.

Reproduced with permission of the copyright owner. Further reproduction prohibited without Dermission. 
(c) Ultrasound: Ultrasound is inexpensive and widely available, and does not expose the patient to ionizing radiation; however, its use is limited because ultrasound waves do not readily pass through bone tissue. It is particularly valuable in the imaging of soft-tissue structures and tendons associated with the joint. Although ultrasound cannot evaluate bone, it can distinguish between solid and cystic soft-tissue lesions. It is also helpful in demonstrating joint effusions behind the knee (Baker's or popliteal cyst) and to guide a needle for aspiration of fluid (arthrocentesis).

(d) Radionuclide Scintigraphy: Also known as a bone scan, this is a sensitive, but nonspecific, method of detecting OA activity. It creates images by scanning affected areas of the body with a gamma camera after a bone-seeking radiopharmaceutical is injected. Radionuclides, such as technetium-99 HDP, are attracted to areas of bone with relatively high blood flow, edema, and high bone-mineral turnover rates (abnormal joint physiology). Joints that are unusually active "light up" in the resulting images. Scintigraphic imaging can be used to distinguish between joints with active disease and those that are altered, but inactive. Moreover, scintigraphic imaging may detect the presence of $O A$ before these changes are detectable on X-rays. Unfortunately, this technique is very costly and time consuming, and requires the use of a radioactive material, which in turn needs special handling. Thus, it is not an appropriate technique for the routine diagnosis or management of $O A$.

(e) Magnetic Resonance Imaging (MRI): MRI is a very expensive imaging technique that uses the behavior of atomic nuclei in a magnetic field to create images. Patients are exposed to radiowaves and a strong magnetic field, rather 
than $\mathrm{X}$-rays or other types of radiation. This technique provides excellent reconstructed images of interior organs, and can delineate muscle, bone, blood vessels, and nerves. MRI is limited as a diagnostic modality because of its expense. Although it is not routinely performed in patients with OA, MRI is useful when other entities, such as meniscal tears or ligament injuries need to be eliminated because it permits the simultaneous imaging of all joint components with excellent contrast between different tissues. MRI has proven to be particularly valuable in imaging joint effusions, menisci, joint ligament, osteophytes, and defects in bone, articular cartilage, and synovial tissue. Moreover, this technique has specific advantages in diagnosing osteonecrosis, internal joint derangements, bone and joint infections, and disorders of the structures around joints (e.g., rotator cuff lesions).

(f) Computed Tomography: Computed tomography or computerized axial tomography is an examination that uses X-ray to obtain a cross sectional image of the human body. CT Scan is helpful in detecting fine detail of cortical bone. Relative to X-ray, CAT reconstructs multiple, two dimensional images. It can distinguish normal and abnormal structures accurately. It is also a relatively expensive test. Relative to MRI, it gives poor definition of soft-tissue boundaries.

\subsection{An Introduction to Knee Sound Signals}

In this work the vibration or sound signals emitted by knee joints during the course of normal movement known as VibroArthroGraphic (VAG) signals has been studied. It 
is hypothesized that this signals are associated with specific pathological conditions. In early stages of this work, the researchers [5] found out two types of knee sound signals.

1. Physiological PatellofemoralCrepitus (PPC) is the vibration signal produced during slow motion (less then $5 \% / \mathrm{s}$ ), which can be measured by Vibration arthrometry (VAM). and

2. The signal produced from the knee when the movement is pretty fast (more than $67 \%$ ), is called "Vibration signal in rapid knee mc ion".

A noninvasiv a procedure to diagnose cartilage pathology would be extremely useful in screening symptomatic patients before knee surgery and in follow-up after surgery. With appropriate techniques for recording and analysis, computer-aided analysis of VAG signals should provide quantitative indices representing the functional state of articular cartilage.

VAG signal has the potential to be widely used non-invasive diagnosis and can be used as an inexpensive diagnosis tool for monitoring of cartilage disorders.

\subsection{Objective of this project}

- The key objective of this work is to find out an inexpensive diagnostic tool for finding the indication of $\mathrm{OA}$ in early stage.

- Another motive of this project is to find out the appropriate angle of the damage area. 


\subsection{Organization of the Report}

- In Chapter 2 we will talk about all the related works. people studied VAG signals in different way, only those comparable with our strategy have been presented here.

- In Chapter 3, I would like to discuss about the methodology such as Adaptive segmentation technique, that I have used in this work as well as the other strategies that people have taken for doing the adaptive segmentation for nonstationary signals

- Chapter 4 will give an idea of the pattern extraction and classification techniques as well as discussion about the Linear Discriminant Analysis (LDA) technique which I have used to do the signal classification

- Finally in Chapter 5 we will discuss the results and also try to give some future direction of this work 


\section{Chapter 2}

\section{Related Works}

\subsection{Previous Research}

Robert Hooke is the person who worked first (in $17^{\text {th }}$ century) in this area and find out that joint noise could be used a diagnostic sign in patients suffering from painful joints [5]. Mollan and his co-workers in Northern Ireland published a series of papers on various aspects of knee joint vibration analysis. They performed experiments on the type of recording system to be used for recording the knee joint vibration signals. A group led by Mang in Germany has also published a paper on the analysis of knee joint sound signals [1]. Based upon the division of sound peaks into frequency groups, this term was apparently able to identify by sound analysis clinically and operatively demonstrated chondromalacia patella, meniscus lesici s, and arthritis. Chondromalacia literally means "softening of the cartilage", and Patellae means "the knee-cap". So Chondromalacia patellae means "softening of the articular cartilage of the kneecap." The articular cartilage is the cartilage lining under the knee-cap that articulates with the knee joint. Under normal circumstances, it is smooth and shiny, so that it glides smoothly along the articular groove of the femur as the knee bends. When 
it "softens", it may break down, causing irregularities along the undersurface of the patella.

In 1985 Mollan [5] and his group published a paper named "What is physiological patello-femoral crepitus?" They basically introduced the PPC signal. They used this name to the fine creaking sensation, which can be felt on all normal patellae as the knee moves slowly. This produces a very regular, reproducible signal. While working they do simultaneous recordings, from accelerometers taped to upper and lower poles of the patella. And they found out the traces are mirror image of each other.

In 1990 [6] the same group made other publications describing the technique of vibration arthrometry being developed to assist the clinical examiner in identifying these vibrations and to improve diagnostic accuracy. They found out that varying the investigative procedure can affect the magnitude of some parameters of the vibration signal. And they do the research for the effect of speed of joint movement and that has been quantified in a pilot study involving 24 patients with internal knee damage. Custom designed hardware was used to measure joint speed as the rate of change of joint angle, which was measured by an electrogoniometer. It was found that the energy content of the vibration, reffected by the peak amplitude and root mean square value was strongly affected by joint speed.

In 1992 a group led by Rangayyan, [1], in the University of Calgary has done a series of research on the analysis of knee vibration signal using Linear Prediction. This paper investigates the possibility of developing a noninvasive method based on analysis of vibration produced by the knee joint. Particularly they used the method of modeling by linear prediction for adaptive segmentation and parameterization of knee vibration signal. Parameters extracted from the LP model, such as dominant poles 
were found to be useful in constructing feature vectors. Dominant poles represent peaks of signal segments in the spectral domain. Two-dimensional feature vectors were constructed for the signal segments analyzed by using the first dominant pole frequency and the ratio of power in the $40-120 \mathrm{~Hz}$ band to total power.

In 1992, a comparative study of simultaneous Vibromyography and electromyography with active human quadriceps has been done Rangayyan and Zhang [12]. Here they compared the Vibromyograghic (VMG) signals and Electromyography (EMG) signals and find out that VMG is a better way to understand the neuromuscular control than using EMG. In contrast to EMG signals, the VMG appears to more directly reflect contractile properties of muscles and may therefore be used also as an aid to the understanding of the neuromuscular control. Vibromyographic (VMG) signals are the low frequency vibration signals generated during muscle contraction and electromyographic (EMG) signals recorded simultaneously during isometric contraction of the human quadriceps muscles. In this paper, they compared VMG with EMG and found out that VMG have diagnostic potential in pediatric muscle diserses and like the EMG signal, exhibits a feature of joint angle dependents. The comparison was accomplished by evaluating the averaged root mean squared (rms) value, mean frequency (MF) and peak frequency (PF) of the VMG and EMG signals for four muscle contraction levels at joint angles of $30^{\circ}, 60^{\circ}$ and $90^{\circ}$. Furthermore the study indicates that the averaged $\mathrm{NF}(6-24 \mathrm{~Hz})$ and PF $(9-19 \mathrm{~Hz})$ of the VMG signals are much lower than the $\mathrm{MF}(75-109 \mathrm{~Hz})$ and $\mathrm{PF}(40-80 \mathrm{~Hz})$ of the EMG signal, but they have similar slopes for $60^{\circ}$ and $90^{\circ}$ joint angle. This means VMG can reflect the same muscle activation patterns like EMG, while the difference in the frequency content between the VMG and EMG reflect the morphological differences between the mechanical and

Reproduced with permission of the copvriaht owner. Further reproduction brohibited without nermission. 
electrical responses of muscle activation.

In 1994, Zhang and Rangayyan [3], published another paper, which mentions about the filtering technique of the VAG signal for muscle contraction interference. While measuring the knee signal using a sensor, they contain significant interference caused by muscle contraction (MCI) that required for the knee movement. Previously a simple frequency domain high-pass filtering technique was used for minimizing the MCI cancellation, but in this work they used an adaptive MCI cancellation as an alternative for MCI cancellation in VAG signal. They basically used the Least Mean Square (LMS) for performing this job. And also they divide the whole MCI reduction in two parts:

1. For detecting and characterizing the correlated MCI components for use as a reference signal in adaptive filtering and

2. Optimizing the step size of the non-stationary adaptive filter

Simon and Verstraete in 1995 did some prediction of knee joint torque from the activity of knee flexion/extention [11]. In this study the authors investigated the relationship between the torque production of the knee flexors and extensors and the EMG of five inuscles crossing the knee. EMG activity and the knee joint torque were recorded for five subjects using surface electrodes and BIODEX system (the Biodex. Multi-Joint System allows the clinician to test, rehab, and analyze a wide range of joint movements at various speeds and various resistance settings. The Biodex System's wide range of operational modes provides optimal flexibility, and is able to safely accommodate all levels of patients from the weakest individual to the strongest athlete), respectively. Data were normalized and averaged for three trials, for each

Reproduced with permission of the copvriaht owner. Further reproduction prohibited without permission. 
of four speeds. The result showed consistent patterns for the torque produced by the muscles and the muscle activity, although minor variations existed. A relationship between torque and EMG was developed for both extension and flexion with squared values ranging from 0.73 to 0.98 .

In 1996, Ragayyan and Moussavi did some progress [4] and published a work on Screening and Adaptive Segmentation of Vibroarthrographic Signals, This paper is basically the continuation of the work reported by Thavathia [1] by using the LP modeling with a constant width reference window ( $\mathrm{N}$ samples).If a sudden variation appears in the middle of a test window then Thavathia's [1] method will show it either at the beginning of the window or at the end of the window, not in the exact position of the variation. So they present here a new adaptive segmentation based on Recursive Least Squares (RLS) algorithm. No reference window is required in this procedure. The filter used in this method tries to predict the signal from a few past samples of the signal in memory, and computes the prediction error. If a change in signal statistics occurs, the tap-weights and the prediction error will show a change as well, which can be detected by applying a threshold.

The method was evaluated with 46 VAG signals, and the segment boundaries computed. Results of application of this method of two VAG signals representative are shown in Figure 2.1. The segment lines are shown by the vertical dashed lines. It is seen from the figures that the clicks (sudden variations) as well as the transitional variations (gradual changes) have been cletected successfully. Comparison of the spectra of consecutive segments from a number of VAG signals indicated that the method is able to track spectral changes very well.

Reoroduced with Dermission of the convriaht nwner. Fluther renrodulation nrohihited withnut nermiccinn 

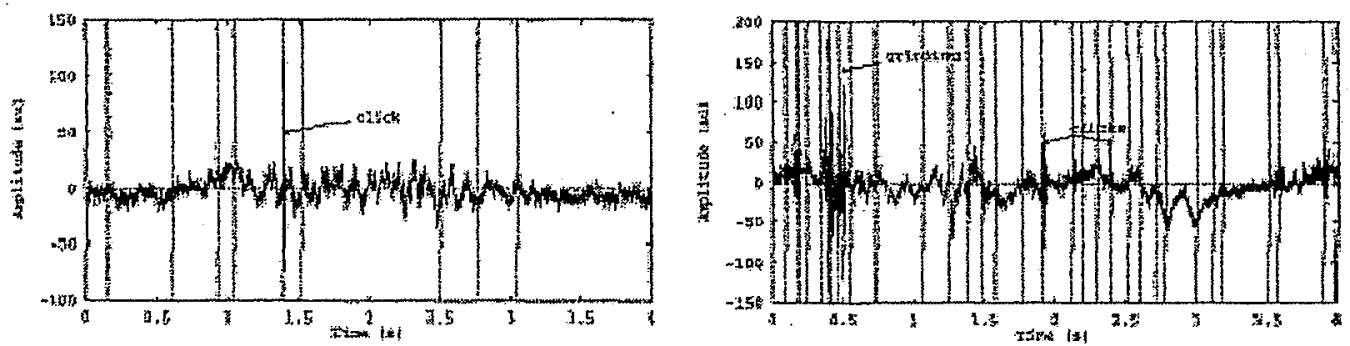

Figure 2.1: the left one shows The arrow points to a click Heard at $20^{\circ}$ extension $0^{\circ}$ is the Fully extended position and the right one shows the Arrows point to clicks and grinding sounds heard during auscultation [4]

In 1996, Kshirsagar, Robson and Herrod [10] worked on the thickness of articular cartilage, which can be an important parameter to assess the progression of osteoarthritis. So in this paper the authors give a complete computerized method for doing the analysis of Magnetic Resonance Imaging (MRI) for directly visualizing the cartilage. Here are the steps they have taken for doing this automation.

- Identify the position of the femoral bone in the 2D MR image

- The coarse edge detection, in order to locate the femur, that edge map is cross related to the standard template of human femur bone to maximize the correlation value

- The image is zoomed to define the area of interest. An edge map is obtained with a finer filter to delineate precisely the edges of the cartilage around the femur

- Eliminate the artifact edges from the trabecular structure, then the thickness, defined as the perpendicular distances between the cartilage edges, is measured 
along the femoral contour at every point and the plot of cartilage thickness in $\mathrm{mm}$ is plotted against the distance along femur

This automated method for cartilage thickness using MRI provides objective and rapid measurements at statistical significant number of points along the joint.

In 1998, Cashman and Kitnev [9] showed up with VAG analysis using MR images of the knee, This paper talking about something related to our study but it really not of our interest. The authors here trying to highlighted the information in the MR slice images of early knee joint damages. The reason for working on it cause, when a patient with osteoarthritis considering for the orthopedic knee surgery, it would be an advantage to present anatomical CT or MRI information to the surgeons in a more accessible format allowing them to display and manipulate anatomical structures as $3 \mathrm{D}$ objects. So the authors trying to develop techniques for automated segmentation and rendering of the major joint structures. They have used $1.5 \mathrm{~T}$ gee body scanner and obtained sagittal T1-weighted spin echo images from 4 volunteers and 2 patients with OA. Segmentation was performed using MIDUS (Medical Information Display and Analysis System: Imperial College).

In recent work, Ching, Jiang and Lee in their paper on Vibration arthrometry, talked about the patients with failed total knee replacement [7]. Here they did the preliminary study on VAM, of artificial knee joint in vivo. As discussed in Section 1.4, basically there are two speed protocols in knee kinematics: i) $2^{0} / \mathrm{s}$, the signal is called "Physiological Patellofemoral Crepitus (PPC)" in which the value of Root Mean Square (rms) is used as a parameter, and ii) $67^{\circ} / \mathrm{s}$, is called "Vibration signal in rapid knee motion" in which Autoregressive(AR) modeling is used for adaptive segmentation and extracting the dominant pole of each signal segment to calculate 
the spectral por ?ios in $\mathrm{f}<100 \mathrm{~Hz}$ and $\approx 2500 \mathrm{~Hz}$.

The study hai $;.$ ected 14 Failed Total Knee Replacement (FTKR) and 12 Normal Total Knee Replacement (NTKR) patients. FTKR is clinically divided into three categories: metal wear, polyethylene wear of the patellar component, and no wear but with prosthesis misalignment. From the study they found out that PPC signals are only detected in two cases of the Metal wear FTKR group. All other NTKR control group, the polyethylene wear FTKR group and the no wear FTKR group have no PPC signals produced. Therefore PPC signals can only be utilized to judge whether there is the metal wear in knee prosthesis, but cannot be used for the detection of polythene wear in the early stage. On the other hand they showed that the vibration signal in rarid knee motion could be used for effectively detecting polythene wear of the patellar component in the early stage.

In another paper by Jiang and Yuan the authors utilize the PPC signals to evaluate the condition of patellar-femoral joint for OA knee patients [8].

PPC signals measured when the knee joint goes through a flexion-extension motion slowly at an angular speed of about $2^{0} / \mathrm{s}$. Typically, a PPC signal is a sequence of vibration pulses generated between the patellar and femoral surfaces. Therefore, a PPC signal usually carries information reflecting the integrity and lubrication of the articular cartilage. Also they analyzed that PPC signal is non-stationary in nature because of its manifestation of slip-stick friction. So to process a PPC signal, they divide it into locally.stationary segments for using signal processing techniques such as AR modeling. They divide osteoarthritis into three types: Type1: the cartilage of patellar-femoral joint is intact, the osteoarthritis found in femoral-tibia joint surface, Type2: degeneration occurs in the surface cartilage of both the femoral-tibial joint 
and femoral trochlea, but not on the patellar surface, Type3: both patellar-femoral and femoral-tibial joint have osteoarthritis.

The results obtained with PPC signals are as follows:

- The proposed method is efficient for the analysis of the Condition of patellar femoral joint cartilage, VAM could be seen as an alternative way of noninvasive diagnosis of $O A$

- By Using the Intraclass Distances it is possible to differentiate OA type 1 alone from OA types 2 and 3

\subsection{Summary of Reviews}

Various works have been done on VAG signals till to date. People applied many adaptive signal processing techniques such as time-frequency analysis, wavelet analysis, stochastic point process modeling for better analysis. But, VAG, as an nonstationary signal, should get more attention in the process of segmentation before it get analyzed. This project mainly devoted for segmenting the signal intelligently, so that after analysis it can give the better indication of the pathological condition and can do the localization as well. 


\section{Chapter 3}

\section{Segmentation Based Analysis of VAG}

Vibroarthrographic (VAG) signals that is emitted from knee when the leg is in motion can be recorded, and could be analyzed for different medical purposes. Here in this work VAG signals has been used which was recorded while knee moves from $135^{\circ}$ to $0^{0}$ position. This kind of movement causes the joint surfaces to rub against one another. The regions of the joint surfaces coming in contact are different at each angle position during the swing. Also the quality of the joint surfaces coming in contact may change with joint angle. This means that the signals are expected to have different characteristics at different joint angles. Therefore the signal produced by a swing is non-stationary in nature, i.e., the statistical characteristics changes with time (angle). All the traditional spectral estimation methods require the signal to be stationary in nature, and further, ergodicity is assumed to permit signal analysis based on single observation. In order to use these techniques on VAG, the signals need to be segmented in such a way so that each part of the signals can be assumed as stationary in nature. Such segmentation can be done in two ways:

- Fixed Segmentation 


\section{- Adaptive segmentation}

Fixed Segmentation: In this process the signal is segmented by a number of segments and the length of each segment will be same. This is commonly used in speech signal processing for short time analysis. This method has also been applied to heart sound analysis. The main problem with fixed segnentation is that sometimes it causes redundant data, which is costly and not meaningful all the time.

Adaptive Segmentation: The main objective of adaptive segmentation is to trace the nonstationarities in the signals that are being processed. So the signal boundaries are determined adaptively and their length depends on the non-stationarity of the signal. This process has been used for analysis of biomedical signals, such as Electroencephalogram (EEG).

\subsection{Different Techniques of Adaptive Segmenta- tion}

People did adaptive segmentation of signals in many research. One such Approach is Wavelet Packet Decomposition (WPD). This approach tries to find the most efficient partition of the frequency axis. The resulting frequency partitioning has narrow bands around prominent (high-energy) harmonics, and has wide bands to span the low-energy noise-like portions of the signal. WPD uses the so-called Best-Basis-Search algorithm (BBSA) to achieve this goal. This algorithm recursively splits the signal into high and low frequency portions and compares these partitions to select the best set that covers the whole frequency range. A drawback is that WPD is only adaptive to the frequency characteristics of the signal, not to its time/dynamic characteristics. 
In a similar way, time adaptive approaches have been developed, but these approaches lack frequency adaptivity.

Gonon proposes an approach to signal analysis that is adaptive to both the time and frequency characteristics of the signal. The key idea is to use separate criteria for the time and frequency segmentations and the combine the two criteria. A useful concept based on the entropy is LSEC (Local Stabilized Entropy Criterion). It is a quantity that represents how the entropy of a windowed part of the signal relates to the entropy of two smaller windowed parts of the signal. Gonon chose first to split the signal into different frequency bands, and then perform a CELS segmentation on each of the bands separately.

Lazareck and Moussavi adaptively did some similar segmentation to our work, they segment the normal swallowing sound signals [15]. It is speculated that the swallowing sound, non-stationary by nature, may be decomposed into char.acteristic segments, representing different stages of swallowing mechanism. They measured the variance for each swallow using a $25 \mathrm{~ms}$ window . A threshold greater that $\mu+\sigma$ of the calculated variance dimension was used to detect the peak of the variance dimensions, which defined the segment boundaries of the swallowing sound. Lastly, each swallowing sound segment was labelled qualitatively as click or non-click segment.

Ahmed [16] also present a novel algorithm for adaptive fuzzy segmentation of MRI data and estimation of intensity inhomogeneties using fuzzy logic. They used a different approach to segmeni images with intensity inhomogeneities is to simultaneously compensate for the shading effect while segmenting the image. This approach has the advantage of being able to use intermediate information from the segmentation while performing the correction. 
The whole project can be summarized in the following way

- Initial segmentation of VAG signals

- Model based adaptive segmentation

- Finding the model order for AR modeling

- Feature extraction, finding the LPC coefficients of the adaptive segments

\subsection{Initial Segmentation (Fixed Width)}

Figure 3.1 is showing the knee signal when the patient is moving his/her leg from $135^{\circ}-0^{0}$ extension. For the sake of simplicity while describing the whole procedure the same signal will be used. The signal is represented in time domain. The signals were sampled at $25 \mathrm{KHz}$ and two sets of data is shown in the graphs.

Initially for doing the fixed segmentation the ratio was around $17: N$, where $N$ is the length of each segment. So for the first half of the signal whose length was 3750 , each segment length was 150 , so the total no of initial segments was 25 (3750/150) for each VAG signal. So initially the total no of segments was $30 \times 25=7500$ for 30 VAG signals, which was used in this experiment for doing the LPC modeling. After that we find out the LP coefficient for each segment. Figure 3.2 shows the initial segment for a signal (the same one shown in Figure 3.1), which has been recorded from a person who is having the knee problem.

In the Figure the lines are showing the fixed segmentation where the number of segments are 25, and each segment of width 150 . The main problem with fixed segmentation is the fixed window constraint. The next section will show how the 


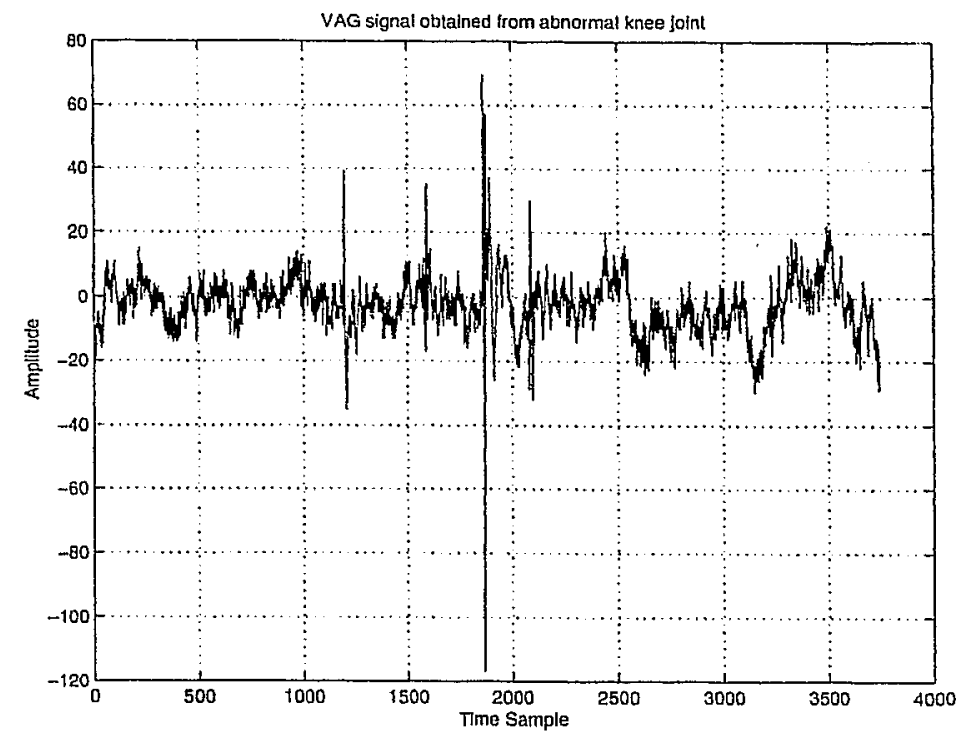

Figure 3.1: VAG when a person moving his leg from $135^{\circ}-0^{\circ}$ extension

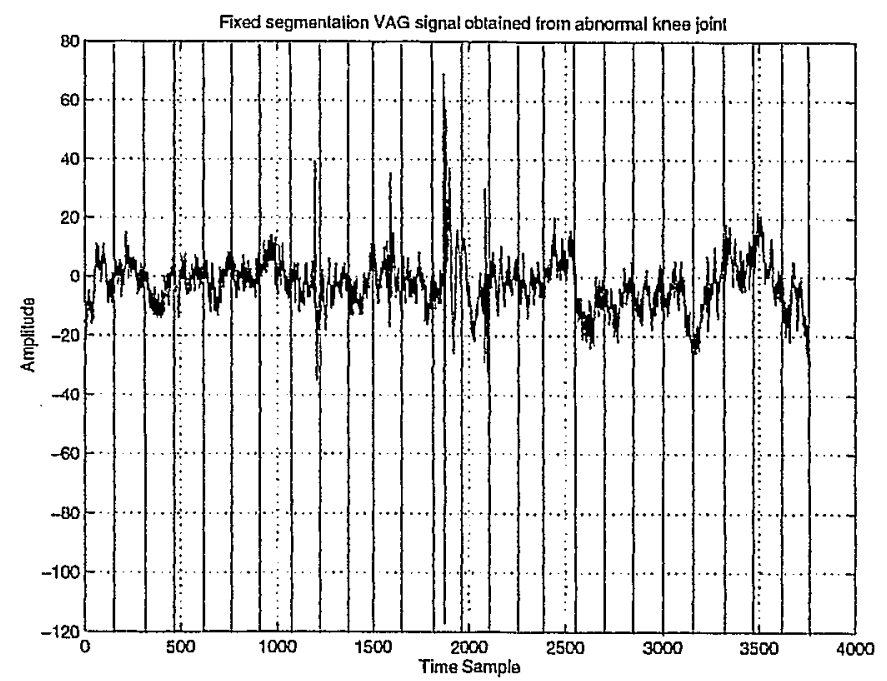

Figure 3.2: Fixed segmentation of the signal shown in Figure 3.1

Reproduced with permission of the copyriaht owner. Further reproduction prohibited without permission. 
proposed algorithm will adaptively reduce the number of segment without losing much information, which is needed for diagnosis.

\subsection{Model-Based Adaptive Segmentation of VAG Signals}

As we know VAG is a non-stationary signal. So usually segmentation has been done on this type of signal to analysis the signal assuming that the signal is stationary for that particular segment. The work in VAG signal processing carried out in such a way, that it can be used as an alternative way to get a precise indication of OA. The main approach to overcome the problem called the fixed window constraint. Analysis of non-stationary signals is usually carried out by means of time segmentation; the size of the segments is generally fixed, and it is based on some a priori modelization of the data. By relaxing most of these modeling assumptions and by allowing for a more general approach to signal segmentation, significant improvements can be achieved.

While analyzing each VAG signal, at first approach the segments was same in lerigth, but after finding out the LPC and choosing a minimum threshold the segments has been rebuilt. Basically, the length of signal has been decided according to the dynamics or the statistical changes occurring in the signal. As we know abnormal knees have rougher cartilage surfaces than normal knees hence VAG signals emitted from knees should have different frequency dynamics compared to the signal from normal knee. So in the proposed segmentation strategy it is hypothesized that the signal from abnormal linee will be having more number of segments with smaller length and on the other hand the signals from normal knees should have less no of 


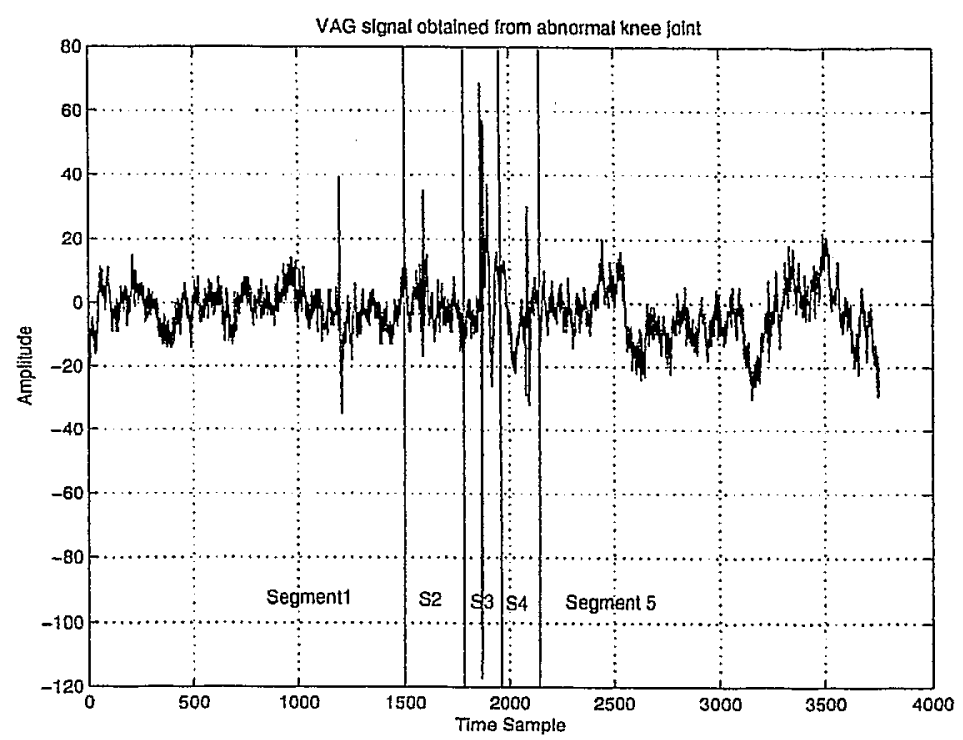

Figure 3.3: Adaptive segmentation of the signal

segments with higher segment length. Furthermore redundant segmentation could be been avoided using the proposed methodology.

This is the most challenging part of this project. After getting the initial fixed number of segments. LPC coefficients for each of the segments are computed and the Euclidean distance between each adjacent segment has been calculated. If the distance is less than a certain "min_dis" value (which is usually less than 1) then those two segments has been added and assumed them as a single segment. the adaptive segmentation algorithm can be summarized as follows:

Here is the pseudo-code (stepwise written) of the algorithm that has been used in this work:

Step1: Reading the Signal in Time Domain 
- Total length of the signal is 7500

- Half of the signal has been used for analysis, cause they are symmetric (1:3750)

Step2: Doing the Fixed Segmentation

- Divide each half of the signal in 25 segments $(3750 / 25)$

- So the length of each segment is now 150

- Find out the LPC Coefficient of each segment

- The model order was 17 so each segment has 17 coefficient right now

- Store all the segments and coefficient in a Cell for total 30 signals

\section{Step3: Doing the Adaptive Segmentation}

- Define a threshold called min_dis, if the minimum distance between two segments is less than that min_dis(this value has been selected adaptively), then the segments will be considered as a single segment

- Check the distance between the adjacent segments

- If the distance is less than min_dis then add them up and assume them as a single segment

- Otherwise keep the old segment as it is

\section{Step4: Finding the LPC Coefficients}

- So finally I got the adaptive segments for each signal, which is a lot less than the fixed segments number

Reproduced with permission of the copyright owner. Further reproduction prohibited without nermission. 
- Find out the LPC coefficients for each segments

- Store all the adaptive segments and their coefficients in a different Cell

- These coefficients have been used as the variables while doing the classifications

For the simplicity the result of adaptive segmentation has been shown on the same signal tha' has been used in Figure 3.2. As it can be seen from Figure 3.3, after each signal gone through the proposed algorithm number of segments for that signal has been reduced remarkably. For the signal under illustration where the number of segments was 25 while doing the fixed segmentation and after going through the proposed adaptive the number of segments becomes 5. From the Figure this is also notable that for stationary part the number of segments are of higher width whereas for the non-stationary parts the segments became smaller in width.

\subsection{Finding the Model Order}

In this phase appropriate model order has been calculated. For maintaining the accuracy instead of taking one single signal, a number of signals (approximately 10 signals has been averaged) has been taken into account. First each VAG signals transferred into frequency domain and then the average of those signals has been calculated. Figure 3.4 is showing the average of $10 \mathrm{VAG}$ signal in FFT form. And from that averaged FFT signals, number of peaks has been calculate when the peaks are going above a certain threshold. Here one more thing has to be mentioned, while calculating the number of peaks the first half of the signals has been taken into account, cause as we know from earlier knowledge that FFT gave us a symmetric representation for first half and the second half of the signal. So after counting the 


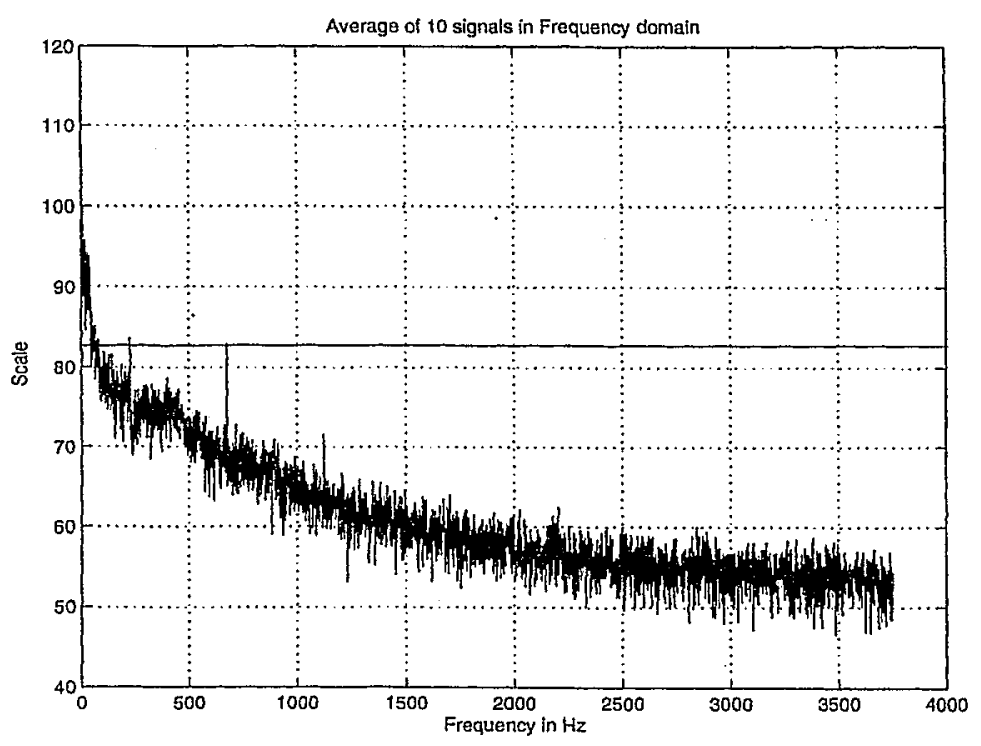

Figure 3.4: Averaged spectrum for finding the number of peaks

number of peaks for one side it has been doubled up and for getting the final order number a threshold value has been added with that.

The threshold line has been has been adjusted adaptively. Finally good result found out when the threshold value has been fixed at 0.75 times of the maximum peak value. So the number of peaks has been calculated whenever the signal crosses this threshold value. In this project the model order was 17 that has been calculated using the above method.

\subsection{LPC Modeling of the Signal}

After getting the final segmentation of the VAG signal, LPC modeling has been performed on each segments, which has to be analyzed. The basic procedure is to 
estimate the power spectral density (PSD) of the signal for each particular segment. The length of the segment is allowed to increase, as long the PSD is same for that part. The basis of LPC modeling is based on AR modeling and is explained as follows

Based on the AR modeling, assume that the current signal (emitted from the knee) sample satisfies the following equation.

$$
x(n)=-\sum_{\ell=1}^{M} a(\ell) x(n-\ell)+w(n) .
$$

Where $M$ is the order of AR model for the stationary random signal $x(n), a(\ell), \ell$ $=1,2, ., M$, are the AR parameters, and $w(n)$ is some input, in the case of knee joint vibration signals, $w(n)$ is totally unknown. From (3.5.1), the approximation of $x(n)$ can be given by

$$
\hat{x}(n)=-\sum_{\ell=1}^{M} a(\ell) x(n-\ell) .
$$

From (3.5.1) and (3.5.2), the resulting approximation error between $x(n)$ and $\hat{x}(n)$ is given by

$$
e(n)=x(n)-\hat{x}(n)=x(n)+\sum_{\ell=1}^{M} a(\ell) x(n-\ell) .
$$

The corresponding mean square error for $x(n)$ is the expectation of the squared error given by

$$
M S E=E\left\{|e(n)|^{2}\right\}
$$

Hence the optimal AR parameters can be found by minimizing (3.5.4) from (3.5.1), the transfer function $H(z)$ of the AR model is given by 


$$
H(z)=\frac{1}{1+\sum_{\ell=1}^{M} a(\ell) z^{-l}}
$$

It follows from (3.5.5) that the Power Spectral Density (PSD) of the model signal is given by

$$
P(f)=\left|\frac{1}{1+\sum_{\ell=1}^{M} a(\ell) e^{-j 2 \pi f l}}\right|^{2}
$$

For processing and analyzing VAG signals, AR modeling technique can be utilized on locally-stationary segment to find the corresponding AR parameters $a(\ell)$ 's. the corresponding PSD for each signal segment is computed from (3.5.6).

The poles of the transfer function $H(z)$ given by (3.5.5) contain useful spectral features of the modelled signal and the dominant poles of the AR model represent the dominant peaks of the signal's PSD. To analyze the VAG signals, the dominant poles can be utilized to show the maximum peak of the signal's PSD.

The AR parameters of a VAG signal could also be used to determine the separability of the signal segments obtained from adaptive segmentation. Let $a_{k}(1), a_{k}(2), . . a_{k}(M)$ be the AR parameters obtained from the $k^{\text {th }}$ signal segment.

So using the above strategy, 17 coefficients [note the model order is 17 in this project] has been found for each segment, which was fed to the SPSS pattern recognition system based on variables. Appendix A provided the table for the coefficient. The segments were labelled as normal and abnormal segment depending on the clinical report obtained. LPC coding of VAG signal has been performed, the coding benefits of adaptive time segmentation and model selection have been tested.

Rebroduced with bermission of the convriaht owner. Further rebroduction nrohibited withol it nermission 


\section{Chapter 4}

\section{Pattern Classification}

Automated methods for making decisions based on inputs play a very important role both in engineering applications and in helping us understand how biological systems responds to their environments. As many engineers and cognitive scientists will attest, the term "input" and "decision" for this pattern classification problem are not clearly defined in theory. In practice the problem is usually decoinposed through design and analysis. A very simple method for making hard decision is the nearest neighbor classifier. This classifier keeps a database of labelled training pattern. Given a test pattern, the nearest neighbor classifier outputs the class of pattern in its database that is "closest" to the test pattern. Any decision metric may be used, but typically Euclidean distance or one of its generalizations are used.

Typically the pattern classification problem can be improved in two techniques:

- Employ various multivariate data analysis techniques (e.g., linear discriminant analysis, neural network, decision trees, support vector machines), and compare them by assessing their model performance. The most accurate model is then used for prediction.

- Keep the multivariate analysis technique fixed and instead work on improving 
the feature representation, by either selecting the most relevant features, or by constructing new features.

In this project the second technique was consideration cause, Most classification algorithms are highly sensitive to the quality of the feature representation. And by selecting the most relevant features, data distribution is transformed to a form amenable to current classification techniques. The feature has been extracted using LP modeling and extracted LPC features for each segment has fed to a linear discriminant analysis (LDA) technique for VAG signal classification.

\subsection{Different Pattern Classification Techniques}

In this section we will briefly discuss the different pattern classification technique that is being in use now a days for different application. The next section we will discuss on Linear Discriminant Analysis (LDA) elaborately cause we use LDA as a pattern classification technique in this project.

\subsubsection{Principal Component Analysis}

The principal component analysis [22] or Karhunen-Loeve transform is a mathematical way of determining that linear transformation of a sample of points in $\mathrm{N}$ dimensional space which exhibits the properties of the sample most clearly along the coordinate axes. Along the new axes the sample variances are extremes (maxima and minima), and uncorrelated. The name comes from the principal axes of an ellipsoid (e.g., the ellipsoid of inertia), which are just the coordinate axes in question. 
By their definition, the principal axes will include those along which the point sample has little or no spread (minima of variance). Hence, an analysis in terms of principal components can show (linear) interdependence in data. A point sample of $\mathrm{N}$ dimensions for whose $\mathrm{N}$ coordinates $\mathrm{M}$ linear relations hold, will show only $(\mathrm{N}-\mathrm{M})$ axes along which the spread is non-zero. Using a cutoff on the spread along each axis, a sample may thus be reduced in its dimensionality. The principal axes of a point sample are found by choosing the origin at the center of gravity and forming the dispersion matrix.

$$
t_{i f}=(1 / N) \sum\left[\left(x_{i}-\left(x_{i}\right)\right)\left(x_{i}-\left(x_{j}\right)\right)\right]
$$

where the sum is over the $N$ points of the sample and the xi are the $i^{\text {th }}$ components of the point coordinates. The principal axes and the variance along each of them are then given by the eigenvectors and associated eigenvalues of the dispersion matrix.

\subsubsection{Genetic Algorithm}

Genetic algorithm neural network (GANN) [21] is often able to provide good answers to questions in the biomedical sciences. It is a procedure that may be used to search among sets of clinical variables for those that are the best predictors. When a neural network uses a genetic algorithm for training there is an increase in computational time, but compared with simple gradient descent, optimization does not fall into local minimum and be more accurate in prediction. A GANN was used to include those predictor variables which contain information to the predictive process. The probability that a variable would be selected was recalculated after each generation in their method. Because the outcome is also a binary indicator. 


\subsubsection{Logistic Regression}

The method of logistic regression addresses the task of concept learning. A linear model of the following form is constructed:

$$
Y=b_{0}+b_{1} X_{1}+b_{2} X_{2}+\ldots \ldots \ldots \ldots b_{k} X_{k}
$$

where $\mathrm{Y}$ is the logit tranformation of the probability $\mathrm{p}$.

The logit transformation of the probability of a value is defined as

$$
Y=\log \left(\frac{p}{(1-p)}\right)
$$

where $\mathrm{p}$ is the probability of an outcome.

The linear function can also be written as a prediction of the probability of a value, e.g.

$$
P(\text { class }=\text { pos })=\frac{1}{1+e^{a+b_{l} x_{1}+b_{2} x_{2}+\ldots \ldots \ldots \ldots . . . b_{k} x_{k}}}
$$

The constant $a$ and the weights $b_{1} \ldots \ldots b_{n}$ are chosen by a regression method so that the predictions for the class are optimal for a given set of classified examples. A number of tools are available for computing the weights.

\subsubsection{Maximum Likelihood Estimation}

There is nothing visual about the maximum likelihood method - but it is a powerful method and, at least for large samples, very precise. Maximum likelihood estimation begins with writing a mathematical expression known as the Likelihood Function of the sample data. Loosely speaking, the likelihood of a set of data is the probability 
of obtaining that particular set of data, given the chosen probability distribution model. This expression contains the unknown model parameters. The values of these parameters that maximize the sample likelihood are known as the Maximum Likelihood Estimates or MLE's.

\subsubsection{Decision Trees}

Binary trees give an interesting and often illuminating way of looking at data in classification problems. In last few decades, considerable research has been conducted on the use of decision trees to solve classification problems. One important feature of decision trees is their capability to break down a complex decision-making or classifying problem into a set of simplified problems. The purpose of a decision classifier is to draw a conclusion through the breaking down and solving of these simple problems that achieves the desired solution of the original complicated problem To construct a decision tree, the tree is first grown to completion so that the tree partitions the training sample into terminal regions of all one class. Tree construction uses the recursive partitioning algorithm, and its input requires a set of training examples, a splitting rule and a stopping rule.

\subsubsection{Linear Discriminant Analysis}

The main idea of this work is to classify between the abnormal and normal patients and we use Linear Discriminant Analysis (LDA) for classifying the data. Linear Discriminant Analysis easily handles the case where the within class frequencies are unequal and their performances has been examined on randomly generated test data. As we know the Vibroarthrographic (VAG) signals i.e., the signal emitted from knee 
while it is moving, is a nonstationary signal like all other biological signal. The method LDA maximizes the ratio of between class variance to the within class variance in any particular data set, thereby guaranteeing maximal separability. So LDA should successfully classify between the normal and abnormal patients analyzing the VAG signals. This technique has been applied for speech recognition as well as for image analysis and showed good results.

Linear discriminant analysis (LDA) is a classical statistical approach for classifying samples of unknown classes, based on training samples with known classes. There are many possible techniques for classification of data. Principal Component Analysis (PCA) and Linear Discriminant Analysis (LDA) are two commonly used techniques for data classification and dimensionality reduction. The prime difference between LDA and PCA is that PCA performs feature classification while LDA performs data classification. PCA changes both the shape and location of the data in its transformed space whereas LDA only provides more class separability by building a decision region between the classes. Linear Discriminant Analysis easily handles the case where the within-class frequencies are unequal and their performances has been examined on randomly generated test data. This method maximizes the ratio of between-class variance to the within-class variance in any particular data set thereby guaranteeing maximal separability.

So the goal of LDA is

- To perform dimensionality reduction while preserving as much of the class discriminatory information as possible.

- It seeks to find directions along which the classes are best separated.

It does so by taking into consideration the two approaches to LDA 
Class-dependent Transformation: Class-dependent LDA produces one transformation per class to discriminate that class from all other classes. This type of approach involves maximizing the ratio of between class variance to within class variance. The main objective is to maximize this ratio so that adequate class separability is obtained. The class-specific type approach involves using two optimizing criteria for transforming the data sets independently.

Class-independent Transformation: Class-independent LDA produces a single transform to maximally separate all classes. This approach involves maximizing the ratio of overall variance to within class variance. This approach uses only one optimizing criterion to transform the data sets and hence all data points irrespective of their class identity are transformed using this transform. In this type of LDA, ea.ch class is considered as a separate class against all other classes.

\section{Mathematical Operations}

For ease of understanding, in this section, the mathematical sperations has been done using LDA will be analyzed for a two-class problem. Each data set has 100 2-D data points. The operations are showing in steps here:

Step 1:. Suppose there are two classes. Class 1 and Class 2. Let $\mu_{1} \mu_{2}$ are the mean vector of class 1 and class 2 respectively. $\mu_{3}$ is the of the entire data set. It has been calculates as

$$
\mu_{3}=p_{1} \mu_{1} p_{2} \mu_{2}
$$

where P1 and P2 are the apriori probabilities of the classes. In the case of this simple two class problem, the probability factor is assumed to be 0.5 .

Step 2: In LDA, within-class and between-class scatter are used to formulate criteria 
for class separability. Within-class scatter is the expected covariance of each of the classes. The scatter measures are computed using Equations 3 and 4.

$$
S_{w}=\sum_{j} p_{j} \times\left(\operatorname{cov}_{j}\right)
$$

Therefore, for the two-class problem,

$$
S_{w}=0.5 \times \operatorname{cov}_{1}+0.5 \times\left(\operatorname{cov}_{2}\right)
$$

All the covariance matrices are symmetric. Let $\operatorname{cov}_{1}$ and $c o v_{2}$ be the covariance of set 1 and set 2 respectively. Covariance matrix is computed using the following equation.

$$
\operatorname{cov}_{j}=\left(X j-\mu_{j}\right) \times\left(X j-\mu_{j}\right)^{T}
$$

The between-class scatter is computes using the following equation.

$$
S_{b}=\sum_{j}\left(\mu_{j}-\mu_{3}\right) \times\left(\mu_{j}-\mu_{3}\right)^{T}
$$

Note that $S_{b}$ can be thought of as the covariance of data set whose members are the mean vectors of each class. As defined earlier, the optimizing criterion in LDA is the ratio of between-class scatter to the within-class scatter. The solution obtained by maximizing this criterion defines the axes of the transformed space. However for the class-dependent transform, the optimizing criterion is computed using equations (5) and (6). It should be noted that if the LDA is a class dependent type, for $L$-class separate optimizing criterion are required for each class. The optimizing factors in case of class dependent type are computed as 


$$
\text { criterion }_{j}=i n v\left(\operatorname{cov}_{j}\right) \times S_{b}
$$

For the class independent transform, the optimizing criterion is computed as

$$
\text { criterion }_{j}=\operatorname{inv}\left(S_{w}\right) \times S_{b}
$$

Step 3: For any L-class problem we would always have $L-1$, non-zero eigen values. This is attributed to the constraints on the mean vectors of the classes in Equation 2. Having obtained the transformation matrices, we can transform the data sets using the single LDA transform or the class specific transforms which ever the case may be.

For the class dependent LDA,

$$
\text { trnasformed_set_j }=\text { transform, } j^{T} \times \text { set }_{-} j
$$

For the class independent LDA,

$$
\text { trnasformed_set }=\text { transform_spec }{ }^{T} \times \text { data_set } t^{T}
$$

Similarly the test vectors are transformed and are classified using the Euclidean distance of the test vectors from each class mean. for proper classification. In this example the classes were properly defined but cases where there is overlap between classes, obtaining a decision region in original space will be very difficult.

Step 4: Once the transformations are completed using the LDA transforms, Euclidean distance or RMS distance is used to classify data points. The Euclidean distance matric is the simplest and most commonly used distance metric for classification of data. It is assumed that we can define a representative number for every 
class involved in the classification problem. The test sample then assigned the class to whose representative number is the closest. The classes may have significant overlap in the input space making the Euclidean distance metric ineffective. To counter this problem data is transformed to a feature space where the classes are better separated or the features are independent and the distance metric is more effective. Euclidean distance is computed using Equation 11 where $\mu_{\text {ntrans }}$ is the mean of the transformed data set, $n$, is the class index and $\mathrm{x}$ is the test vector. Thus for $\mathrm{n}$ classes, Euclidean distances are obtained for each test point.

$$
d_{i s t \_n}=(\text { transform_n_spec })^{T} \times x-\mu_{n t r a n s}
$$

The smallest Euclidean distance among the distances classifies the test vector as belonging to class.

\subsection{Pattern Analysis of VAG}

LDA helps us to discriminate between the normal abnormal signals as two separate classes based on linear classification rule. Here is a flow chart showing how LDA worked on our systen. Here is a complete description of each block of the flow chart which will basically give a overview of the whole procedure that has been taken for doing this model based segmentation of VAG signal analysis.

Input Signal: $30 \mathrm{VAG}$ signals have been used as the input signal. 16 signals have been taken from normal knee joint and 14 signals from abnormal knee joint.

Adaptive Segmentation: Each signal has been adaptively segmented depending on the dynamics of the signal characteristics. After segmenting 30 signals, a total of 71 


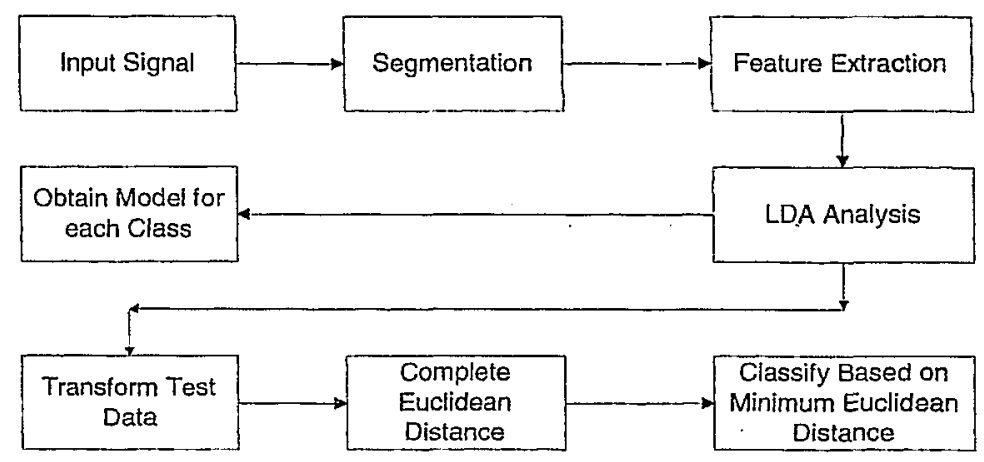

Figure 4.1: Flowchart of VAG Analysis

segments have been produced which has been used as for analyzing the VAG signals.

Feature Extraction: After getting the segments the features has been extracted from that segments using Autoregressive modeling. In this part of the work a total of 17 coefficients were produces for each segments.

LDA Analysis: After getting the features, which are the LPC coefficients in this project, the transformation matrix is formed by the eigenvectors corresponding to the dominant eigenvalues of the optimizing criterion. This LPC coefficients worked as the variables to the linear discriminant analysis tool for classification of VAG signals.

Training and Testing: This part has been done by the SPSS system which has been used for discriminant analysis of the VAG signals. So the system got trained with the coefficient used for representing each segment. For testing the system "Leave-oneout" strategy has been taken, where one of the input signal assumed to be unknown and it has been classified based on the training set. After transforming the test data, it computes the Euclidean distance between the test vector and trained vector and perform the classification based on the Euclidean distance. 


\section{Chapter 5}

\section{Results and Conclusion}

\subsection{Motivation for Our strategy}

The main motivating factor for this project is to develop a non-invasive diagnostic tool using VAG signal for detecting some pathological condition such as Osteoarthritis. For fulfilling that objective a model based segmentation and analysis has been done on the knee signals; which is called as VAG. The propose scheme should have the following criteria

- The diagnosis should use advanced digital signal processing techniques, so it should have the ability to correctly diagnosis OA in early stage.

- The process designed to an inexpensive way of diagnosis.

- It should correctly localize the problem area (the angle where the surface is more rough)

- The whole system set-up should have simple mechanism. 


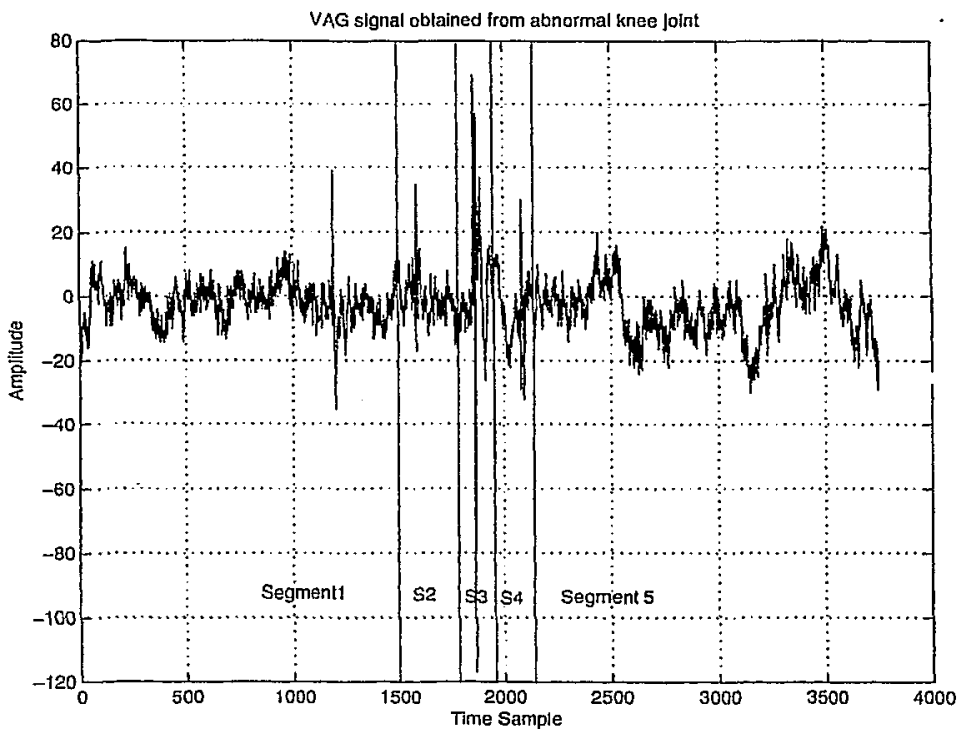

Figure 5.1: Adaptive segmentation of the signal showing the localization

\subsection{Results of Localization of Pathology}

In this section it has been explained how the proposed scheme can analyze the nonstationarity and can do the localization of a VAG signal. For describing the procedure it is necessary to repeat the same figure that has been used in Chapter 3 when adaptive segmentation procedure was described. As the project is basically doing the model based segmentation, where the main concept is to segment the signal effectively which will correctly diagnosis and do the localization. This is a segmented form of a abnormal knee signal after doing the segmentation using the proposed model based segmentation.

After getting the adaptive segmentation using our model approach, we will get a scenario such as one in Table 5.1. The table is showing the width of each segment as 


\begin{tabular}{|c|c|c|}
\hline No of Segments & Starting Point & Ending Point \\
\hline 1 & 1 & 1500 \\
2 & 1501 & 1800 \\
3 & 1801 & 1950 \\
4 & 1951 & 2100 \\
5 & 2101 & 3750 \\
\hline
\end{tabular}

Table 5.1: Starting and End point of each segment after doing the adaptive segment well as the starting and ending point for that particular segment. The above figure represents a total 5 segments of different width depending on the characteristics of the VAG signal.

It can be clearly seen from Figure 5.1 that the segments are localizing the problem area. The results of the localization are as follows:

- It can be notified from the table the segment length gets bigger when the signal is pretty stationary and its getting thinner when the signal become more nonstationary.

- As it has been described in Chapter 3 the adaptive segmentation has been done on the signal after they segmented in a fixed width.Features have been extracted for each fixed width segment and distance between the segments has been calculated.

- A threshold value was adjusted adaptively and if the distance between two adjacent segment is less than the threshold, then they assumed to be a single segment. This way the non-stationary part of the signal can be recognized, cause more non-stationary the signal, more number of segments will be obtained.

- The localization can be more precise when the threshold is minimized between the adjacent segments. But on the other hand when threshold become lower 
the number of segment for each signal will be increased. So cost for analyzing for more number of segments will be higher.

\subsection{Results}

The analysis was done on 30 VAG signals obtained from subjects both normal and are from patients presenting with symptomatic knee. Among those 16 signals are from the abnormal knee joint and the rest of the signals are from normal knee joint. After doing the adaptive segmentation for each VAG signal, each one was having a different number of segments. A total of 71 segments have been used finally for analysis. Each segment has been named either as "Normal" or "Abnormal" segment as per the clinical report observed through arthroscopy. For analysis each segment has been classified in two categories, "1" for the segments from the normal VAG signal and "2" for the segments from the abnormal VAG signal. After doing the segmentation, features has been extracted from each segment using the AR modeling to obtain the LPC coefficients. The filter order has been selected using the criteria described in chapter 3, and the resulted order was 17 . So each segment has 17 features, which was used as the variables while using the SPSS system for LDA pattern analysis. At the starting of doing the Discriminant analysis using SPSS software, it finds out the mean and standard deviation of all the variables. Then the LDA analysis has been performed. As shown in Table 5.2, 32 segments out of 71 were classified in category1 and the rest of the segments were in category2.

Reproduced with permission of the copyright owner. Further reproduction prohibited without permission. 


\begin{tabular}{|c|c|c|c|}
\hline Actual Group & No of Segments & \multicolumn{2}{|c|}{ Predicted Group } \\
\cline { 2 - 4 } & & Normal & Abnormal \\
\hline Normal & 32 & 28 or $87.5 \%$ & 4 or $12.5 \%$ \\
\hline Abnormal & 39 & 7 or $17.9 \%$ & 32 or $82.1 \%$ \\
\hline Total & 71 & Overall Accuracy & $84.5 \%$ \\
\hline
\end{tabular}

Table 5.2: Classification results using LDA

An acceptable number of segments have been experimented and the classification result suggests that VAG signal could be used as a diagnostic tool for detecting OA. The number and percentage of cases correctly classified and misclassified are tabulated. Out of 32 Normal segments 28 or $87.5 \%$ has been classified properly whereas 4 or $12.5 \%$ segments have been misclassified. In other words, out of 32 normal segments the system detects 28 segments as normal but 4 segments have been misclassified and detected as abnormal segments. For the case of 39 abnormal segments, 32 or $82.1 \%$ have been correctly classified i.e., detected as abnormal and 7 or $17.9 \%$ got misclassified. So the overall accuracy of the classification become $84.5 \%$.

For the classification using "Leave-one-out" method, one segment is used as a test vector while the others are used as the trained vector. While analyzing the VAG signals in this strategy, in case of normal 32 segments, 23 or $71.9 \%$ has been corrected classified, while 9 or $28.1 \%$ got misclassified. For analyze the abnormal 39 segments 27 or $69.2 \%$ got correctly classified and 12 or $30.8 \%$ got misclassified. The overall accuracy for this case is $70.4 \%$.

After doing the classification using the segments, it has calculated that how many segments from each signal has been correctly identified. If most of the segments from a particular signal has been classified correctly then its assumed that the signals has been classified correctly. Using that procedure out of 30 signals 26 signals has been

Reproduced with permission of the copyright owner. Further reproduction prohibited without permission. 


\begin{tabular}{|c|c|c|c|}
\hline Actual Group & \multirow{2}{*}{ No of Segments } & \multicolumn{2}{|c|}{ Predicted Group } \\
\cline { 3 - 4 } & & Normal & Abnormal \\
\hline Normal & 32 & 23 or $71.9 \%$ & 9 or $28.1 \%$ \\
\hline Abnormal & 39 & 12 or $30.8 \%$ & 27 or $69.2 \%$ \\
\hline Total & 71 & Overall Accuracy & $70.4 \%$ \\
\hline
\end{tabular}

Table 5.3: Classification using leave-one-out method

classified properly (either normal or abnormal) and 4 signals got misclassified. So the overall accuracy of this work can be said as $86.7 \%$.

\subsection{Conclusion}

In conclusion, 30 signals have been adaptively segmented and analyzed to differentiate between the normal and abnormal knee sounds signal. The segmentation has taken care of the non stationarity of the VAG signal. The segmentation technique has also been used to localize the range of pathology in the signal.

\subsection{Possible Future Work Direction}

Possible future work in this research can be to find out how early we can detect $\mathrm{OA}$ and how these signals can help us to determine the cause of Anterior knee pain. Moreover, PPC is also a non-stationary signal. So STFT or TFD and wavelet analysis can be performed on PPC signals. 


\section{Appendix A}

This is the resulted coefficients that have been extracted from the VAG signals and used for analyzing the signals. A total of 71 segments from 30 signals have been used. The name of the signals has been given in such a way so that it can be easily understood, from which signal the segment has been extracted Seg4.1 mean the first segment from 4 th signal. Total 30 signals have been used which are numbered from signal 2 to signal 32. Start point and end point is showing the segment length. Category ' 1 ' represent that the segment is from the normal VAG signal while category '2' represent that the segment is extracted from a abnormal VAG signal. Coef_1 to Coef 18 are the coefficients that has been extracted as the features of the segments and they are used as the variable for discriminant analysis. 
AR Coefficients of the VAG signals used in this project

\begin{tabular}{|c|c|c|c|c|c|c|c|c|c|c|c|}
\hline No of Seg & Coef 8 & Coef 9 & Coef 10 & Coef 11 & Coef 12 & Coef 13 & Coef 14 & Coef 15 & Coef 16 & Coef 17 & Coef 18 \\
\hline 1 & $1.63 \mathrm{E}-01$ & $-2.52 \mathrm{E}-01$ & $2.29 \mathrm{E}-01$ & $-9.21 E-02$ & $6.94 \mathrm{E}-02$ & $2.48 \mathrm{E}-02$ & $-1.74 \mathrm{E}-01$ & $2.62 E-01$ & $-2.06 \mathrm{E}-01$ & $5.76 \mathrm{E}-02$ & $2.44 \mathrm{E}-02$ \\
\hline 2 & $-2.01 E-02$ & $-9.92 \mathrm{E}-02$ & 5.92E-02 & $1.60 \mathrm{E}-02$ & $-1.44 E-02$ & $2.13 E-02$ & $-4.27 E-02$ & $-4.68 \mathrm{E}-03$ & $2.68 \mathrm{E}-02$ & $-5.55 E-02$ & $4.34 \mathrm{E}-02$ \\
\hline 3 & $8.35 \mathrm{E}-03$ & $-1.10 \mathrm{E}-01$ & $3.74 \mathrm{E}-02$ & $-3.88 E-03$ & $-1.83 \mathrm{E}-02$ & $-4.23 E-02$ & $2.61 \mathrm{E}-02$ & $5.72 E-02$ & $-1.16 E-01$ & $8.66 \mathrm{E}-02$ & $-7.56 \mathrm{E}-02$ \\
\hline 4 & $=-02$ & $4.51 \mathrm{E}-02$ & $1.48 E-02$ & $1.91 E-02$ & $9.96 \mathrm{E}-03$ & $-8.79 E-02$ & $3.00 \mathrm{E}-02$ & $-1.40 E-02$ & $2.68 \mathrm{E}-02$ & $-3.16 E-02$ & $4.52 \mathrm{E}-03$ \\
\hline 5 & $1.40 \mathrm{E}-02$ & $1.29 \mathrm{E}-02$ & $7.12 \mathrm{E}-04$ & $-2.19 \mathrm{E}-02$ & $-4.00 \mathrm{E}-02$ & $3.77 E-02$ & $-8.60 \mathrm{E}-03$ & $-2.31 \mathrm{E}-02$ & $1.10 \mathrm{E}-02$ & $-6.24 E-02$ & $6.31 \mathrm{E}-02$ \\
\hline 6 & $-9.46 E-02$ & $-9.98 \mathrm{E}-03$ & $8.38 \mathrm{E}-02$ & $-4.05 \mathrm{E}-02$ & $-8.13 E-02$ & $4.72 \mathrm{E}-02$ & $4.30 E-03$ & $4.64 \mathrm{E}-02$ & $-1.05 \mathrm{E}-02$ & $-1.33 \mathrm{E}-02$ & $1.36 \mathrm{E}-03$ \\
\hline 7 & $6.13 E-02$ & $-2.96 E-02$ & $-6.13 \mathrm{E}-03$ & $5.85 \mathrm{E}-06$ & $7.48 E-03$ & $-4.07 E-03$ & $-3.05 E-02$ & $-1.72 \mathrm{E}-03$ & 1.72E-02 & $-1.09 \mathrm{E}-02$ & $2.66 \mathrm{E}-02$ \\
\hline 8 & $-2.51 E-02$ & $-3.36 \mathrm{E}-03$ & $9.06 \mathrm{E}-03$ & $-7.96 \mathrm{E}-03$ & $2.83 \mathrm{E}-02$ & $-2.43 E-02$ & $1.90 \mathrm{E}-02$ & $-2.30 \mathrm{E}-02$ & $7.13 \mathrm{E}-03$ & $-1.50 E-02$ & $2.64 \mathrm{E}-03$ \\
\hline 9 & 1.19E-02 & $1.55 E-02$ & $2.92 \mathrm{E}-02$ & $2.92 \mathrm{E}-02$ & $-3.59 E-02$ & $1.79 \mathrm{E}-02$ & $3.85 \mathrm{E}-02$ & $-1.65 E-02$ & $2.17 \mathrm{E}-02$ & $-5.29 E-02$ & $-4.00 E-02$ \\
\hline 10 & $-3.63 \mathrm{E}-02$ & $-5.02 E-02$ & $-2.90 \mathrm{E}-03$ & $2.06 \mathrm{E}-02$ & $4.23 \mathrm{E}-02$ & $-3.37 E-02$ & $-2.72 E-02$ & $2.55 \mathrm{E}-03$ & $5.11 E-02$ & $-1.92 \mathrm{E}-02$ & \\
\hline 11 & $3.54 \mathrm{E}-01$ & $-3.77 E-01$ & $7.39 \mathrm{E}-02$ & $1.44 E-01$ & $-3.32 E-01$ & $2.24 \mathrm{E}-01$ & $-1.74 \mathrm{E}-01$ & $8.01 E-02$ & $-1.04 \mathrm{E}-01$ & $2.40 \mathrm{E}-02$ & \\
\hline 12 & $6.36 \mathrm{E}-02$ & $3.20 \mathrm{E}-01$ & $-3.29 \mathrm{E}-01$ & $-3.02 \mathrm{E}-02$ & $1.22 \mathrm{E}-01$ & $-1.76 E-01$ & $1.38 E-01$ & $-7.70 \mathrm{E}-02$ & $-1.70 E-01$ & $2.70 \mathrm{E}-01$ & $-1.64 \mathrm{E}-01$ \\
\hline 13 & $-1.13 \mathrm{E}-01$ & $1.93 E-01$ & $-1.67 \mathrm{E}-01$ & $2.97 \mathrm{E}-01$ & $-9.87 E-02$ & $1.53 \mathrm{E}-01$ & $-2.04 \mathrm{E}-01$ & $1.49 E-01$ & $-6.93 \mathrm{E}-02$ & $1.79 E-01$ & $-1.21 E-01$ \\
\hline 14 & $2.66 E-02$ & $1.80 \mathrm{E}-01$ & $-2.19 E-01$ & $1.97 E-03$ & $2.22 E-01$ & $-2.39 E-01$ & $3.06 E-01$ & $-2.53 E-01$ & $3.99 E-02$ & $1.06 E-01$ & $-6.70 E-02$ \\
\hline 15 & $-2.18 \mathrm{E}-01$ & $1.41 \mathrm{E}-01$ & $-4.17 E-02$ & $-1.04 E-01$ & $1.64 E-01$ & $-5.34 E-03$ & $-2.45 E-01$ & $1.62 E-01$ & $1.02 E-01$ & $-1.70 E-01$ & $3.38 E-02$ \\
\hline 16 & $3.23 E-02$ & $-5.32 E-02$ & $-3.39 \mathrm{E}-02$ & $-3.27 E-02$ & $8.82 E-02$ & $-3.88 E-02$ & $4.28 E-02$ & $-3.43 E-02$ & $-1.56 E-02$ & $-4.07 E-02$ & $3.21 \mathrm{E}-02$ \\
\hline 17 & $4.43 E-02$ & $-3.41 \mathrm{E}-02$ & $3.92 \mathrm{E}-02$ & $-3.48 \mathrm{E}-02$ & $-1.15 \mathrm{E}-03$ & $-2.88 E-02$ & $2.99 \mathrm{E}-02$ & $-4.72 E-03$ & $3.25 E-02$ & $-3.19 E-02$ & $-1.01 \mathrm{E}-02$ \\
\hline 18 & $5.30 E-02$ & $3.80 \mathrm{E}-03$ & $9.84 \mathrm{E}-02$ & $-1.69 \mathrm{E}-01$ & $-4.72 E-02$ & $2.09 E-01$ & $-1.62 E-01$ & $9.17 E-02$ & $-5.24 E-02$ & $6.82 E-02$ & $-3.60 E-02$ \\
\hline 19 & $-2.43 E-03$ & $-2.96 \mathrm{E}-02$ & $3.75 \mathrm{E}-02$ & $1.16 \mathrm{E}-02$ & $-3.17 E-02$ & $7.52 E-02$ & $-3.73 E-02$ & $-1.71 \mathrm{E}-02$ & $-1.54 \mathrm{E}-02$ & $5.24 \mathrm{E}-02$ & $-3.83 E-02$ \\
\hline 20 & $6.18 \mathrm{E}-02$ & 1.07E-02 & $1.75 \mathrm{E}-02$ & $3.40 E-03$ & $-3.67 \mathrm{E}-02$ & $7.13 \mathrm{E}-02$ & $1.22 \mathrm{E}-02$ & $-7.10 \mathrm{E}-02$ & $-5.40 \mathrm{E}-02$ & $3.02 E-02$ & $-5.92 E-02$ \\
\hline 21 & $-3.36 \mathrm{E}-02$ & $6.32 E-02$ & $1.16 \mathrm{E}-03$ & $3.17 \mathrm{E}-02$ & $-9.47 E-02$ & $1.66 \mathrm{E}-01$ & $-7.14 \mathrm{E}-02$ & $-1.37 E-03$ & $-1.52 E-01$ & $1.69 E-01$ & $-7.93 \mathrm{E}-02$ \\
\hline 22 & $2.58 \mathrm{E}-03$ & $1.67 \mathrm{E}-02$ & $-3.90 \mathrm{E}-03$ & $-5.19 E-03$ & $-3.04 E-02$ & $6.78 \mathrm{E}-03$ & $-2.90 \mathrm{E}-02$ & $5.34 \mathrm{E}-02$ & $-7.10 E-02$ & $4.89 \mathrm{E}-02$ & $-1.23 \mathrm{E}-02$ \\
\hline 23 & $-5.21 E-03$ & $-4.08 \mathrm{E}-02$ & $-1.56 \mathrm{E}-02$ & $-5.49 E-02$ & $5.00 E-03$ & $1.04 E-02$ & $-2.03 E-02$ & $2.48 E-02$ & $-3.52 \mathrm{E}-02$ & $-1.68 E-02$ & $-2.10 \mathrm{E}-02$ \\
\hline 24 & $-1.23 E-02$ & $5.27 \mathrm{E}-02$ & $-4.85 E-02$ & $8.52 \mathrm{E}-02$ & $-5.14 E-02$ & $-3.28 E-02$ & $7.99 E-03$ & $5.40 E-03$ & $-7.51 E-03$ & $-3.64 \mathrm{E}-02$ & $-3.56 \mathrm{E}-03$ \\
\hline 25 & $3.58 \mathrm{E}-02$ & $-1.29 \mathrm{E}-01$ & $1.59 \mathrm{E}-01$ & $-1.19 E-01$ & $2.96 \mathrm{E}-02$ & $-1.17 \mathrm{E}-02$ & $4.57 E-02$ & $-1.04 E-01$ & $3.42 \mathrm{E}-02$ & $-7.03 \mathrm{E}-02$ & $3.16 \mathrm{E}-02$ \\
\hline 26 & $91 \mathrm{E}-02$ & $2.27 E-01$ & $-2.20 \mathrm{E}-01$ & $5.43 E-02$ & $1.08 \mathrm{E}-01$ & $-1.91 \mathrm{E}-01$ & $1.08 \mathrm{E}-01$ & $8.58 E-02$ & $-7.73 E-02$ & $-9.58 \mathrm{E}-02$ & $9.18 E-02$ \\
\hline 27 & $50 \mathrm{E}-01$ & $2.00 E-01$ & $-8.74 \mathrm{E}-02$ & $6.20 E-02$ & $-1.82 E-02$ & $-2.75 E-02$ & $-4.83 \mathrm{E}-02$ & $-1.16 E-02$ & $-7.77 \mathrm{E}-02$ & $-2.86 \mathrm{E}-02$ & $8.24 \mathrm{E}-02$ \\
\hline 28 & $2.63 E-03$ & $-3.66 \mathrm{E}-02$ & $3.91 \mathrm{E}-02$ & $1.12 \mathrm{E}-02$ & $-3.56 \mathrm{E}-02$ & $-1.37 E-02$ & $3.53 \mathrm{E}-02$ & $-8.80 \mathrm{E}-03$ & $-1.92 \mathrm{E}-02$ & $-2.09 \mathrm{E}-02$ & $1.52 \mathrm{E}-02$ \\
\hline 29 & $-3.82 \mathrm{E}-03$ & $-7.97 E-02$ & $-2.35 \mathrm{E}-03$ & $-4.84 E-02$ & $-2.37 \mathrm{E}-02$ & $1.58 \mathrm{E}-02$ & $-6.32 E-02$ & $3.71 \mathrm{E}-02$ & $-2.57 \mathrm{E}-02$ & $-1.99 \mathrm{E}-02$ & $4.54 E-02$ \\
\hline 30 & $-1.84 \mathrm{E}-01$ & $3.59 \mathrm{E}-02$ & $2.04 \mathrm{E}-01$ & $-1.02 E-01$ & $-9.36 \mathrm{E}-02$ & $1.66 \mathrm{E}-01$ & $-1.74 \mathrm{E}-01$ & $4.79 E-02$ & $-7.81 \mathrm{E}-02$ & $1.51 E-01$ & $-8.14 E-02$ \\
\hline 31 & $-3.99 E-02$ & $6.11 \mathrm{E}-02$ & $-1.10 \mathrm{E}-01$ & $-4.45 E-02$ & $-8.03 E-03$ & $7.61 \mathrm{E}-02$ & $2.85 E-02$ & $-9.38 E-02$ & $6.17 \mathrm{E}-02$ & $9.49 \mathrm{E}-03$ & $-6.38 E-02$ \\
\hline 32 & $6.40 \mathrm{E}-02$ & $6.59 E-02$ & $4.35 E-02$ & $2.80 \mathrm{E}-02$ & $5.66 \mathrm{E}-03$ & $5.96 \mathrm{E}-03$ & $7.07 \mathrm{E}-03$ & $1.19 E-02$ & $1.31 \mathrm{E}-02$ & $4.19 \mathrm{E}-03$ & $-8.30 E-03$ \\
\hline 33 & & $-4.44 \mathrm{E}-03$ & $4.19 E-03$ & $1.03 E-02$ & $-3.55 \mathrm{E}-02$ & $-3.20 E-02$ & $1.27 \mathrm{E}-02$ & $-8.71 E-03$ & $2.69 \mathrm{E}-02$ & $-2.06 E-02$ & $5.77 \mathrm{E}-03$ \\
\hline 34 & $16 \mathrm{E}-01$ & $2.18 \mathrm{E}-02$ & $-8.21 \mathrm{E}-02$ & $1.11 \mathrm{E}-01$ & $-5.13 \mathrm{E}-02$ & $-2.54 \mathrm{E}-02$ & & $-9.69 E-02$ & $4.06 \mathrm{E}-02$ & & $-5.28 \mathrm{E}-02$ \\
\hline 35 & $-1.08 \mathrm{E}-02$ & $-1.74 E-02$ & $-1.18 E-02$ & $-1.91 E-02$ & $-9.41 \mathrm{E}-03$ & \begin{tabular}{|l|}
$-2.26 \mathrm{E}-02$ \\
\end{tabular} & $-2.34 \mathrm{E}-03$ & $2.20 \mathrm{E}-02$ & $-3.20 \mathrm{E}-02$ & $3.70 \mathrm{E}-02$ & $-2.39 \mathrm{E}-02$ \\
\hline 36 & $1.43 \mathrm{E}-02$ & $-8.66 \mathrm{E}-02$ & $1.52 E-02$ & $1.64 \mathrm{E}-03$ & $-5.25 E-02$ & $-1.51 E-03$ & $-3.57 \mathrm{E}-02$ & $4.77 \mathrm{E}-02$ & $8.46 E-03$ & $-1.73 E-02$ & $1-1.02 E-02$ \\
\hline
\end{tabular}




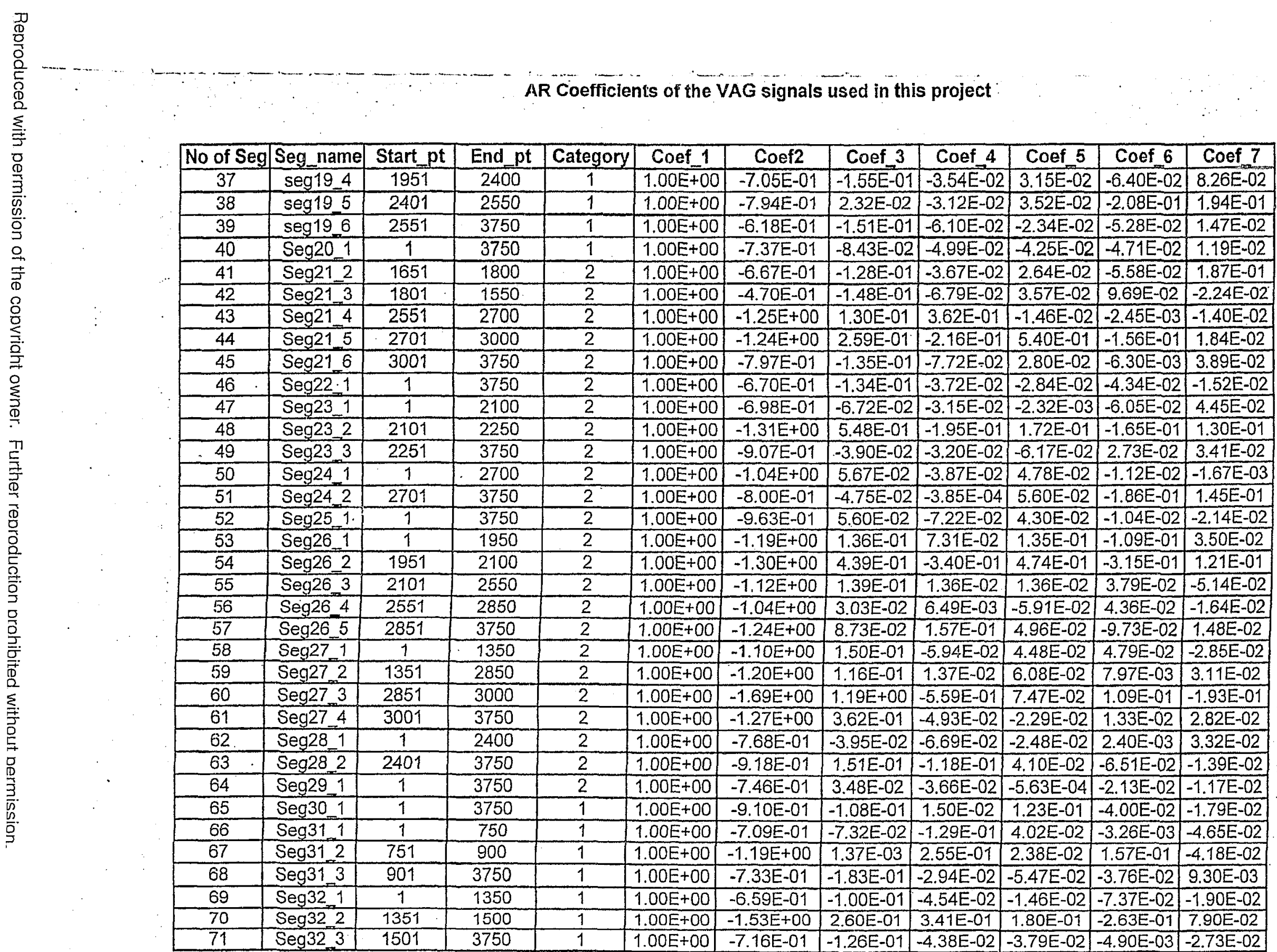




\section{Appendix B}

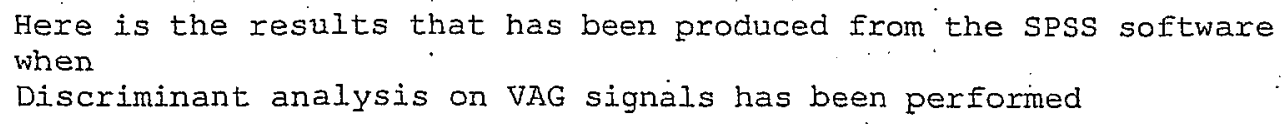

\section{Discriminant}

Notes

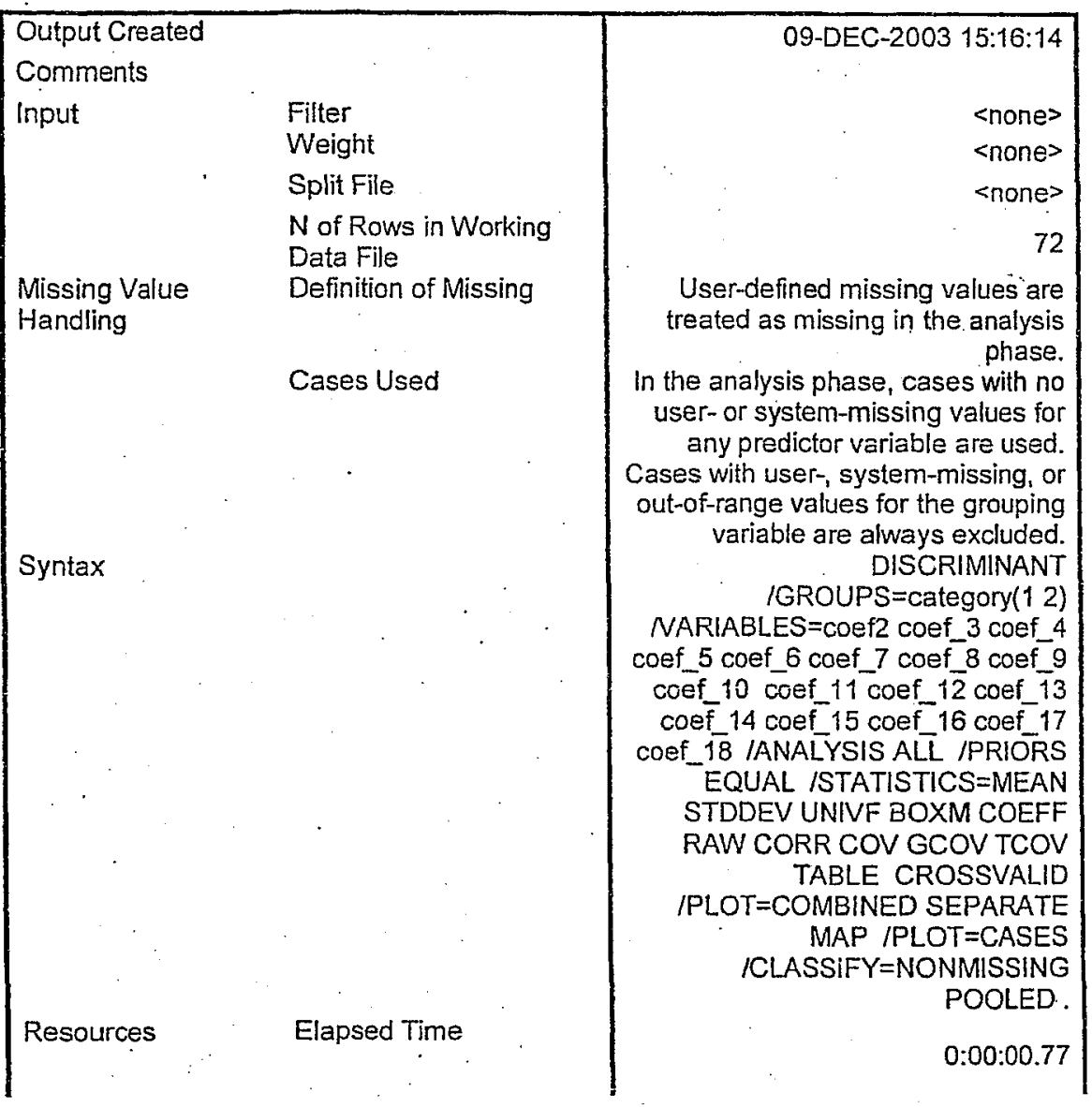




\section{Analysis 1}

\section{Box's Test of Equality of Covariance Matrices}

\begin{tabular}{|l|r|r|}
\multicolumn{2}{|c|}{ Log Determinants } \\
\hline CATEGORY & Rank & $\begin{array}{c}\text { Log } \\
\text { Determinant }\end{array}$ \\
\hline 1 & 17 & -96.386 \\
2 & 17 & -99.790 \\
Pooled within-groups & 17 & -90.942 \\
\hline
\end{tabular}

The ranks and natural logarithms of determinants printed are those of the group covariance matrices.

\section{Test Results}

\begin{tabular}{|ll|r|}
\hline Box's M & & 504.993 \\
& Approx. & 2.417 \\
df1 & 153 \\
df2 & 13517.224 \\
Sig. & .000 \\
\hline
\end{tabular}

Tests null hypothesis of equal population covariance matrices.

\section{Summary of Canonical Discriminant Functions}

\begin{tabular}{|l|r|r|r|r|}
\hline & & & \\
\hline Function & Eigenvalue & $\%$ of Variance & Cumulative \% & $\begin{array}{r}\text { Canonical } \\
\text { Correlation }\end{array}$ \\
\hline 1 & $.883(a)$ & 100.0 & 100.0 & .685 \\
\hline
\end{tabular}

a First 1 canonical discriminant functions were used in the analysis.

Wilks' Lambda

\begin{tabular}{|l|r|r|r|r|}
\hline Test of Function(s) & $\begin{array}{r}\text { Wiks' } \\
\text { Lainbda }\end{array}$ & Chi-square & df & Sig. \\
\hline 1 & .531 & 38.304 & 17 & .002 \\
\hline
\end{tabular}

Functions at Group Centroids

\begin{tabular}{|l|c|}
\hline \multirow{2}{*}{ CATEGORY } & Function \\
\cline { 2 - 2 } & 1 \\
\hline 1 & 1.023 \\
\hline
\end{tabular}

Unstandardized canonical discriminant functions evaluated at group means 


\section{Classification Statistics}

Classification Processing Summary

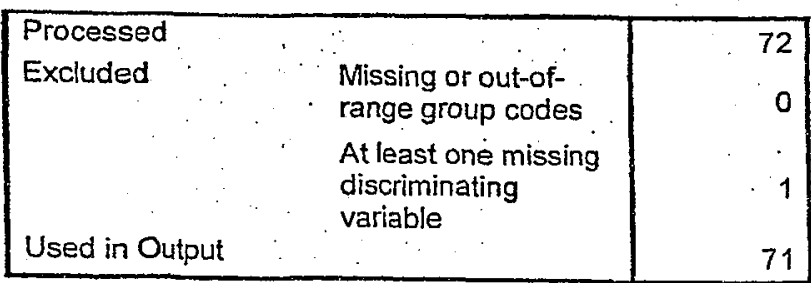

Classification Results(b,c)

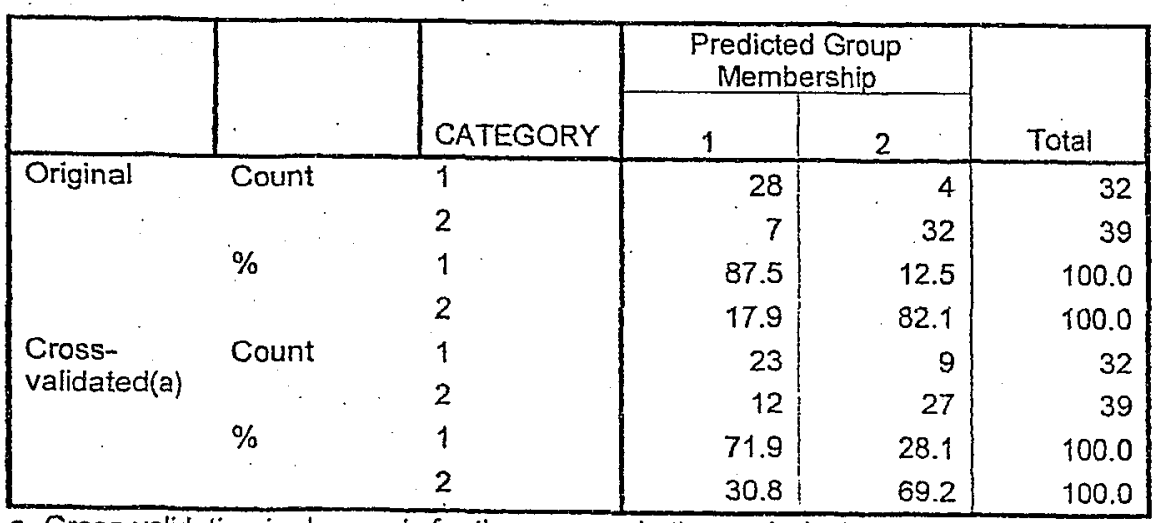

a Cross validation is done only for those cases in the analysis. In cross validation, each case is classified by the functions derived from all cases other than that case.

b $84.5 \%$ of original grouped cases correctly classified.

c $70.4 \%$ of cross-validated grouped cases correctly classified. 


\section{Bibliography}

[1] Sanjeev Tavathia, Rangaraj M. R, G.D.Bell, K.O.Laidly, Y.T. Ahang Analysis of Knee Vibration Signals Using Linear Prediction', IEEE Trans. on Biomed. Eng., Vol. 39, No 9, September1992.

[2] Y.T.Zhang, W.A. Rolston, R.M. Rangayyan, C.B. Frank, G.D. Bell. Wavelet Transform analysis of Vibroarthrographic (VAG) signals obtained during dynamic knee movement Department of Electrical and Computer engineering, Sport Medicine Center, Department of Surgery. The University of Calgary, Alberta, Canada 1993-94.

[3] Zhang, Y.T.; Rangayyan, R.M. Adaptive Cancellation of Muscle Contraction Iinterference in Vibroarthrographic Signals Biomedical Engineering, IEEE Transactions on , Volume: 41 Issue: 2, Feb. 1994 5Page(s): 181 -191

[4] Moussavi, Z.K., Rangayyan R.M., Frank C. et al., Screening and Adaptive Segmentation of Vibroarthrographic Signals IEEE Trans. on Biomed. Eng., Vol. 43 (1):15-23, Jan. 1996.

[5] D. E. Beverland, W. G. Kernohan, and R. A. B. Mollan, What is Physiological Patello-femoral Crepitus? Med. Biol. Eng. Comput., vol. 23, pp. 1249-1250, 1985.

[6] W. G. Kernohan, D. A. Barr, G. F. McCoy, and R. A. B. Mollan, Vibration Arthrometry, in Assessment of Knee Disorder scientific and technical record, August 1990. 
[7] Ching-Chauan jiang, Ju-Hong Lee, Tung-Tai Yuan, Vibration Arthrometry in Patients with Failed Total Knee Replacement IEEE Transactions on Bio Med Engg. Vol 47, No 2, January 2000

[8] Ju-Hong Lee*, Ching-Chuan Jiang, and Tung-Tai Yuan, Vibration Arthrometry in Patients with Knee Joint Disorders IEEE Transactions On Biomedical Engineering, Vol. 47, No. 8, August 2000

[9] R.I. Kitney, P.M.M Cashman, M.E. Carter, Fast Automated Segmentation and Visualization Methods for MR Image of the Knee Joint in Arthritis Proceeding of the 20 th annual international conference of the IEEE engineering in medicine and biology society, Vol 20, No2, 1998.

[10] Kshirsagar, A.; Robson, M.D.; Watson, P.J.; Herrod, N.J.; Tyler, J.A.; Hall, L.D.; Computer Analysis of MR Images of Human Knee Joints to Measure Femoral Cartilage Thickness Engineering in Medicine and Biology Society, 1996. Bridging Disciplines for Biomedicine. Proceedings of the 18th Annual International Conference of the IEEE, Volume: 2 , 31 Oct.-3 Nov. 1996 Page(s): $746-747$ vol.2

[11] Simon, B.N.; Verstraete, M.C.; Mulavara, A.P.; Zehner, L.; Reisberg, S.; Prediction of Knee Joint Torque from Muscle Activity during Knee Flexion/extension Engineering in Medicine and Biology Society, 1995. IEEE 17th Annual Conference, Volume: 2, 20-23 Sept. 1995 Page(s): 1311 -1312 vol.2

[12] Y.T. Zhang, C.B. Frank, R. M. Rangayyan, G.D. Bell, A Comparative Study of Simultaneous Vibromyography and Electromyography with Active Human Quadriceps IEEE Trans. on Biomed. Eng., Vol. 39, No 10, October1992

[13] Suresh Balakrishnama, Aravind Ganapathiraju, Joseph Picone. Linear Discriminant Analysis for Signal Processing Problems Institute of signal and In formation processing. , 1999 IEEE southeast contest, Mississipi State University. Mississippi, USA. Email: balakrish,ganapath, picone @isip.msstate.edu 
[14] Kil-Moo Lee, Member, IEEE, Peter Meer, Senior Member, IEEE, and Rae-Hong Park, Member, IEEE Robust Adaptive Segmentation of Range Images

[15] Lisa J. Lazareck and Zahra Moussavi Adaptive Swallowing Sound Segmentation by Variance Dimension. Department of Electrical Engineering, University of Manitoba, Winnipeg, Canada, R3T 2N2 E-mail: lazareck@ee.umanitoba.ca, mousavi@ee.umanitoba.ca

[16] M. N. Ahmed, S. M. Yamany, A. A. Farag, and T. Moriarty Bias Field Estimation and Adaptive Segmentation of MRI Data Using a Modifed Fuzzy C-Means Algorithm Univ. of Louisville, E.E. Dep+., Louisville, $\mathrm{KY}$ 40292 E-mail:fmohamed,faragg@cairo.spd.louisville.edu Department of Neurological Surgery, University of Louisville, Louisville, KX 40292

[17] Krishnan, Sridhar Masters Thesis Department of Electrical and Computer Engineering, University of Calgary, April 1996

[18] New York Brace, www.mybrace.com/b-knee-is.html

[19] Arthritis Information Fact Sheet, http://www2.state.id.ns/dhw/arth/factsheet.html

[20] American College of Rheumatolotgy Diagonostic Criteria for OA http://www.mercmedicus.com/pp/us/hcp/diseasemodules/osteoarthritis/diagnosis.jsp

[21] North American Congress on Biomechanics, University of Waterloo, Waterloo, Ontario, Canada, August 14-18, 1998

[22] The Data Analysis Brief Book, http://rkb.home.ch/rkb/littleA.html 